\title{
Mixing in turbulent compressible heated coaxial jets: A numerical study
}

\author{
Riadh Ouzani ${ }^{a, *}$, Sofiane Khelladi ${ }^{b}$, Amélie Danlos ${ }^{b}$ \\ ${ }^{a}$ LESEI Laboratory, Mechanical Engineering Department, University of Batna 2, Algeria \\ ${ }^{\mathrm{b}}$ Arts et Metiers Institute of Technology, CNAM, LIFSE, HESAM University, F-75013 Paris, France
}

\section{H I G H L I G H T S}

- The approach employed is based on Monotonic Integrated Large Eddy Simulation.

- The potential cores length of the coaxial jets depends strongly on the temperature gradients.

- The temperature gradient leads to the rapid development of the inner Kelvin-Helmholtz vortices.

- The turbulent mixing becomes more homogeneous in the case of the non-isothermal jet.

Keywords:

Turbulence

Coaxial jets

Free shear layer

Mixing enhancement

Compressible flows

\begin{abstract}
A B S T R A C T
The computation of compressible coaxial jets has moved into the field of interest not only in fundamental research but also in industrial applications, especially in chemical engineering. Numerical simulations of such flows are performed here, using a code specifically developed for gaseous turbulent flows which can also take into account chemical re-actions. The coaxial jet can be regarded as a model of injection device in industrial ap-plications; one can cite combustion and aeroacoustics technology. Three-dimensional numerical simulations in this configuration, already published by various authors in open literature are limited to incompressible isothermal flow. In our work, we have explicitly taken into account the temperature gradient effects on the dynamics and mixing mech-anisms. Indeed, we have investigated a spatially developing compressible (isothermal and non-isothermal coaxial jet). The numerical model is based on time and space resolutions of compressible Navier-Stokes equations. The piecewise parabolic method (PPM) is combined with a linearized Riemann solver. This scheme adds non-linear dissipation intermittently just where and when needed in order to avoid spurious oscillations and guarantee monotonicity for the advection equation. The simulation can, therefore, be regarded as large-Eddy simulations: large scales are accurately solved with minimal viscosity and non-linear dissipation extracts energy out of the small scales in order to avoid non-physical oscillations. In order to study the mixing between Air-Air flows, we consider the mixture fraction $f$ to track the mixing between two species seeded in the coaxial jets. A great attention is paid to the spatial-temporal evolution of the mixture fraction $f$, with a particular interest to probability density function. The comparison between the numerical results and experimental data is fairly good, with respect to the mean and turbulent fields. We found that the inner potential core length in the non-isothermal configuration reduced with respect to the isothermal coaxial jet due to the gradient of temperature. It was shown that in the non-isothermal coaxial jet, the temperature gradient leads to the rapid development of the inner Kelvin-Helmholtz vortices implying an efficient mixing of thespecies close to the exit of the computational domain.
\end{abstract}

* Corresponding author.

E-mail addresses: ouzani.riad@yahoo.com (R. Ouzani), sofiane.khelladi@ensam.eu (S. Khelladi), amelie.danlos@lecnam.net (A. Danlos). 


\section{Introduction}

Coaxial jet flow has a wide scope of usage in many engineering applications. Particularly, it is broadly used in process with combustion as for instance, gas turbine combustors and combustion chambers of rocket engines [1]. Aerospace applications are another field that coaxial jet is involved, i.e., turbofan engines. In this field of study, using additional jets leads to the reduction of the noise level produced by a single jet $[2,3]$.

Coaxial jets are produced when a fluid stream with velocity $\mathrm{U}_{2}$ issued from an outer nozzle of diameter $\mathrm{D}_{2}$, is added outside a central jet flow with velocity $U_{1}$ issued from the inner nozzle of diameter $D_{1}$ (see Fig. 1).

Numerous studies [4-11] have been devoted to the effects of initial conditions (nozzle geometry, lip thickness, velocity ratio, pressure ratio,...) on flow instability, mixing and turbulence.

A survey of the literature indicates that the rate of momentum transfer between the jets is proportional to the velocity ratio, and the increase of velocity ratio promotes faster mixing.

Improvement of mixing fluid streams flow plays a central role in a large range of technical applications which differ from simple chemical mixtures for jet engines [6-8]. In order to have a better mixing of fluids, nozzles with different shapes and geometry specifications have been used in the wide range of velocity from subsonic to supersonic [9].

Studies regarding the mixing coaxial jet flow are mostly concentrated on mixing behaviour of incompressible flow.
There were several experimental (e.g. Refs. [11-13]), numerical (e.g. Refs. $[7,8,14,15])$ and both experimental and numerical (e.g. Refs. [16-18]) studies in the past demonstrated the use of coaxial jets as a promising technique in enhancing downstream fluids mixing. The effect of inlet velocity ratio on the mixing behaviour of coaxial jets was first studied experimentally by Forstall and Shapiro [19]. They found that near and far fields of mixing coaxial jets are significantly affected by the velocity ratio.

The similarity between mixing subsonic coaxial jet and the single jet was determined by Ko and Kwan [20] and Kwan and Ko [21]. Moreover, these authors have mentioned the existence of two shear layer regions and the formation of two potential cores associated with each region.

Dahm et al. [22] in an experimental study investigated a coaxial jet flow by means of the planar laser-induced fluorescence (PLIF) technique. They analyzed a broad range of vortex patterns and their interaction of near-field mixing. These authors showed also that the instabilities in the inner and outer shear-layers are strongly coupled.

Champagne and Wygnanski [23] showed that the inner potential cone length is strongly dependent on velocities and nozzles surfaces ratios. However, the outer potential core length seems to be independent of velocities ratios and equal to eight times the outer nozzle thickness.

Numerical investigation of incompressible mixing in the coaxial jet has been already considered in widespread works. Ranga Dinesh et al. [14] studied numerically mixing and intermittency in a coaxial turbulent jet. They discussed the effect of vortex shedding on velocity fluctuation inside the inner mixing.

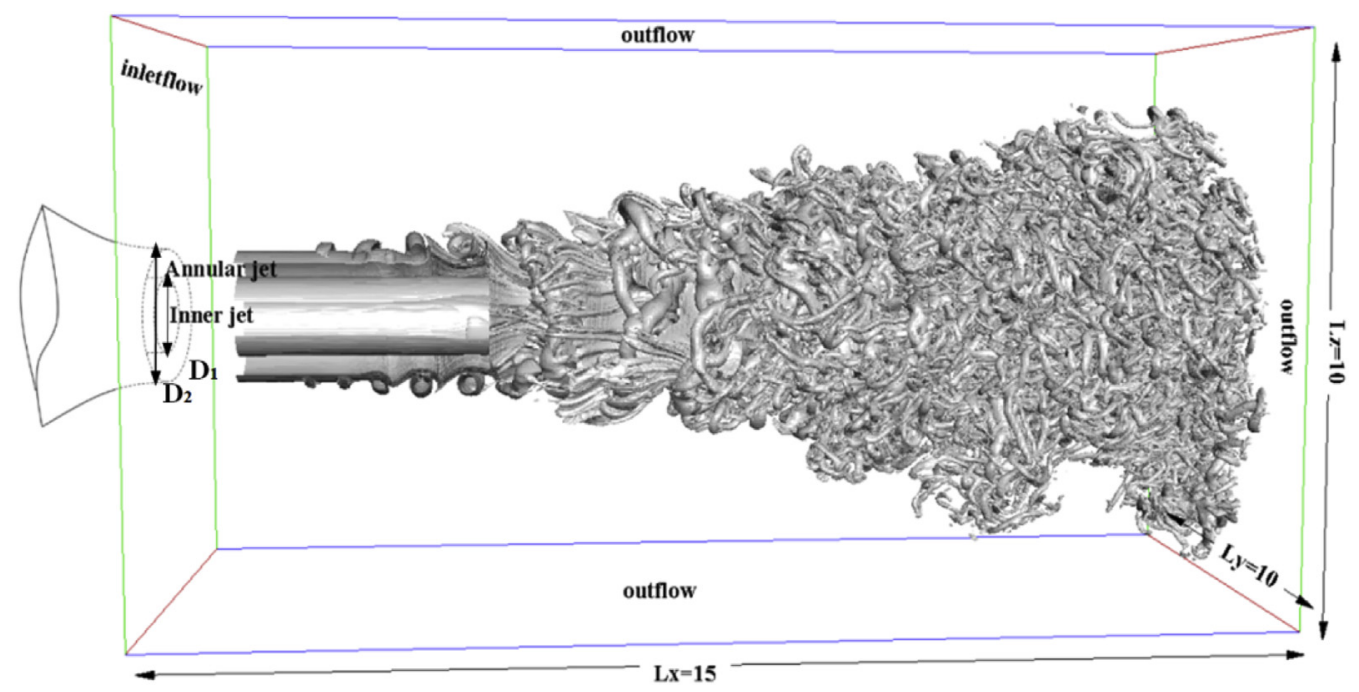

Fig. 1 - Sketch of spatially developing coaxial jets and computational domain with boundary conditions. 
The effect of upstream condition on the incompressible coaxial jet has been already studied both experimentally and numerically. Balarac et al. $[5,7,8]$ numerically investigated the influence of upstream conditions on the mixing behaviour of a high-velocity ratio coaxial. They reported that mixture fraction field and the process of turbulent mixing in coaxial flow pertain on upstream conditions. Balarac et al. [24] in other works studied numerically forced and natural flow of mixing improvement in the coaxial jet. They found that excited flow enhances better the mixing jets compared to the natural one.

Saravanan et al. [25] analyzed numerically the effect of subsonic coaxial jets on potential core length by using a constant Mach number in the inner nozzles and different Mach numbers of annular flow, they concluded that if the velocity of flow between inner and annular nozzle increases, turbulent intensity will increase as well that result in declining in the potential core length.

The compressibility of the flow issuing from two coaxial nozzles has not been yet discussed widely. Reynier and $\mathrm{Ha}$ Minh [26] have performed a standard $k-\varepsilon$ model to simulate air-air compressible, turbulent coaxial jets having a large velocity ratio between outer and inner jets. Their results show a strong instability of the coaxial jet close to the inlet. In the same work, these authors have observed the development of a recirculation region in the near field of coaxial jets due to the higher velocity ratio. They also found that there is a strong coupling between inner and outer shear-layers.

Ouzani and Si-ameur [27] highlighted the role of turbulent structures on the mixing process by using large eddy simulation; they found that the turbulent mixing activity is undergoing to an intermittent specificity of the coherent vortices.

Murakami and Papamoschou [28] investigated experimentally mean flow enhancement of compressible flow in coaxial jets. Noise emission, thermal signature and combustion in high-speed turbulent jets were taken into consideration in their study. They reported that by increasing area of annular jet and Mach number, the inner potential core is extended.

Effect of upstream excited flow on the mixing behaviour was also studied experimentally by Guitton et al. [29,30]. To study the matter, they used a different method to measure the velocity of other needed parameters.

As mentioned above, coaxial jets have been extensively studied. But, these studies mostly focused on incompressible or compressible flow and an upstream excitation separately. However, a deeper physical understanding of the mixing process performed in these flow configurations still requires extensive research efforts.

In fact, very few investigations have considered the impact of temperature gradients on the dynamics of flow and mixing process.

In recent years, the coaxial jet configuration has particularly attracted interest especially in technological combustion systems because it provides better mixing fluids in addition to their simple geometry.

Researches on combustion using burner with coaxial injectors have considerably increased as attested by a significant number of publications [31-36]. These studies dealt with combustion efficiency, flame stabilization and pollutant emissions from coaxial burners.

Tabet et al. [33], showed numerically that hydrogen addition improves fuel and oxidant jets mixing. This mixing quality improvement has a significant effect on the consumption of hydrocarbon and the air driving to the jet center which leads to faster fuels burning and reduce the residence time, length and thickness of the flame. In the same context, Hashemi et al. [34], studied numerically the effect of air turbulence intensity on NOx emission in coaxial burner fueled with hydrogen. They found that increasing air turbulence intensity supply from the outer nozzle leads to a reduction of formation in NO concentration.

From the literatures above on combustion performance and emissions reduction, we can conclude that thorough the knowledge of turbulence-mixing interactions between jets in the coaxial configuration is a prerequisite to improve the control of the flame ignition, pollutant emissions and it also allows for the design and performance enhancement of devices such as combustion chamber, injection systems.

This work presents a numerical simulation of compressible mixing in a coaxial jet with an emphasis on the temperature gradient effect.

Our computational model is three dimensional because flows in most applications are turbulent, involving a wide range of length scales. Detailed information is given about the structure and dynamics of scales by fully solving the compressible Navier-Stokes equations.

In our approach, chemical reactions are avoided in this study, in order to focus on the effect of the turbulent structures on the mixing process to provide complementary information on the mixing in parallel to combustion works.

The remainder of the paper is organized as follows: the next section Governing equations and numerical method gives detailed information about the governing equations of the physical problem, followed with the numerical method used for the computational study. Section Flow and simulation parameters, presents the computational parameters used in this work. In section Results and discussion, the results obtained from numerical simulations of coaxial jets are analyzed and discussed, with emphasis put on comparisons between both configurations (isothermal and non-isothermal). Finally, the conclusion is presented in section Conclusion.

\section{Governing equations and numerical method}

The Numerical method is based on High order Godunov's scheme called PPM (piecewise parabolic method) [37] combined with linearized Riemann solver of Roe [38] to solve the complete compressible Navier-Stokes equations (the equations for conservation of mass, momentum, energy and detailed mass of each species are written in conservative form). The overall scheme is second-order accurate in time. An optimal value of the time step $\Delta t$ is selected at each time $t$. A diffusion-reaction operator is added as a source term in Navier-Stokes equations, and switched on or off depending on the problem under consideration, especially with regard to the type, direct or large eddy, of numerical simulation.

Computations of compressible coaxial jets are performed using a code specifically developed to numerically simulate 
gas flows whose composition may evolve with location $\vec{x}$ and time $t$. It is based on the usual set of gas flow equations, with the vector of the conserved variables:

$U(\vec{x}, t)=\left\{\rho, \rho u_{1}, \rho u_{2}, \rho u_{3}, \rho e, \rho Y_{i}\right\}^{T}$

$\rho$ is the density of both inner and other flow, $\left(u_{1}, u_{2}, u_{3}\right)$ the three velocity components, $Y_{i}$ is the mass fraction of the species $i$. The total energy of the flow is defined by:

$e=\frac{1}{2}\left(u_{1}^{2}+u_{2}^{2}+u_{3}^{2}\right)+\overline{C_{v}} T$

where the average heat capacity per unit mass $\overline{\mathrm{Cv}}$ is $\overline{\mathrm{Cv}}=$ $\sum_{i=1}^{n s} \operatorname{CviY}_{i}\left(n_{s}\right.$ is the number of involved species). Thermodynamics of the gas mixture is modelled with an ideal gas equation. The pressure $P$ and the temperature $T$ are related by:

$\frac{P}{\rho}=\frac{R T}{\bar{M}}$

where $R$ is the universal gas constant and $\bar{M}$ is the average molar mass calculated from the molar mass $M_{i}$ of each species $i: \bar{M}^{-1}=\sum_{i=1}^{n_{s}} Y_{i} / M_{i}$.

Each component of $U(\vec{x}, t)$ corresponds to a conserved quantity like mass, momentum or energy. The total energy $e$ is the sum of the mechanical and internal energies of the gas mixture.

The equations for conservation of mass, momentum, energy and detailed mass of species $i$ are written in conservative form.

$\frac{\partial U(\vec{x}, t)}{\partial t}+\sum_{j=1}^{3} \frac{\partial F_{j}(U(\vec{x}, t))}{\partial x_{j}}=S(U(\vec{x}, t))$

$F_{j}(U(\vec{x}, t))=\left\{\begin{array}{c}\rho u_{j} \\ \rho u_{j} u_{1}+\delta_{j 1} P \\ \rho u_{j} u_{2}+\delta_{j 2} P \\ \rho u_{j} u_{3}+\delta_{j 3} P \\ (\rho e+p) u_{j} \\ \rho Y_{i} u_{j} \\ \ldots\end{array}\right\}$

where:

$$
S(U)=\left\{\begin{array}{c}
0 \\
\sum_{j=1}^{j=3} \frac{\partial\left(\frac{\rho}{\operatorname{Re}}\right) \tau_{1 j}}{\partial x_{j}} \\
\sum_{j=1}^{j=3} \frac{\partial\left(\frac{\rho}{\operatorname{Re}}\right) \tau_{2 j}}{\partial x_{j}} \\
\sum_{j=1}^{j=3} \frac{\partial\left(\frac{\rho}{\operatorname{Re}}\right) \tau_{3 j}}{\partial x_{j}} \\
\nabla \cdot\left(\frac{\overline{C p} \rho}{\operatorname{Pr} \cdot \operatorname{Re}} \nabla T\right)+\nabla \cdot\left(\frac{\rho}{\operatorname{Re}} \vec{u} \cdot \overline{\bar{\tau}}\right) \\
\nabla \cdot\left(\frac{\rho}{\operatorname{Sc} \cdot \operatorname{Re}} \nabla Y_{i}\right)
\end{array}\right\}
$$

The right-hand side term of equation (4) contains terms for viscous stress, scalar diffusivity.

$\tau_{i j}=\frac{\partial u_{i}}{\partial x_{j}}+\frac{\partial u_{j}}{\partial x_{i}}-\frac{2}{3} \delta_{i j} \sum_{K=1}^{K=3} \frac{\partial u_{K}}{x_{K}}$

$\delta$ is the Dirac delta. Re, Pr and Sc are the Reynolds number, Prandtl and Schmidt numbers respectively. $\overline{\mathrm{Cp}}$ is the heat capacity at constant pressure of the mixture per unit mass. The values of $\overline{C p}, \overline{C v}$ and $\bar{M}$ evolve and have to be calculated at each $(\vec{x}, t)$.

The full compressible Navier-Stokes equations (4) are split up in three one-dimensional inviscid operators $L_{j}(j=1,2,3)$ :

$L_{j}: \quad \frac{\partial U(\vec{x}, t)}{\partial t}+\frac{\partial F_{j}(U(\vec{x}, t))}{\partial x_{j}}=0$

and three-dimensional viscous-diffusive operator:

$\psi: \quad \frac{\partial U(\vec{x}, t)}{\partial t}=S(U(\vec{x}, t))$

The fourth-order accurate piecewise parabolic method (PPM) [37] together with the approximate Riemann Solver of Roe [38] solve the inviscid equations (5). The overall scheme is second-order accurate in time. The diffusion terms are regrouped in a source term of Navier-Stokes equations.

The spatial discretization of equation (6) is done by finite volume. The integration in time is accomplished by secondorder accurate low storage Runge-Kutta time stepping [39].

The averaged values $U_{i}$ over the ith grid cell are updated by computed the fluxes through the Riemann Solver between two discontinuities at each cell interface over one timestep.

The space higher-order precision is achieved by taking into account the spatial variation of the discretized function in order to build two states $U_{L}$ (left) and $U_{R}$ (right) as an average of the information which is crossing to the interface over one timestep. The PPM algorithm generates a parabolic interpolation function for the primitive dynamic and scalar fields over each cell. The parabola is defined by its average over the cell and the border values which are computed with a fourthorder differencing formula. They are corrected where they generate new extremema in order to realize monotonicity.

The combined solution is computed as follows:

$U^{n+2}=\psi^{1 / 2} L_{3} L_{1} L_{2} \psi L_{2} L_{1} L_{3} \psi^{1 / 2} U^{n}$

\section{Flow and simulation parameters}

The flow, the physical model and the coordinate system are schematically depicted in Fig. 1. As can be seen from Fig. 1, the coaxial nozzles consist of two main nozzles. The inner or primary nozzle of diameter $D_{1}$ and annular or secondary nozzle with diameter $\mathrm{D}_{2}$.

Morris [40], Michalke and Hermann [41] found that the mean velocity profile shape in the nozzle affects the first instability and the flow features.

The initial condition consists of two hyperbolic-tangent profiles in streamwise direction and zero mean transverse and spanwise velocities as shown in Fig. 2a. A weak white noise $U_{\text {noise }}\left(\vec{x}_{0}, t\right)$ was superimposed to the inflow shear 

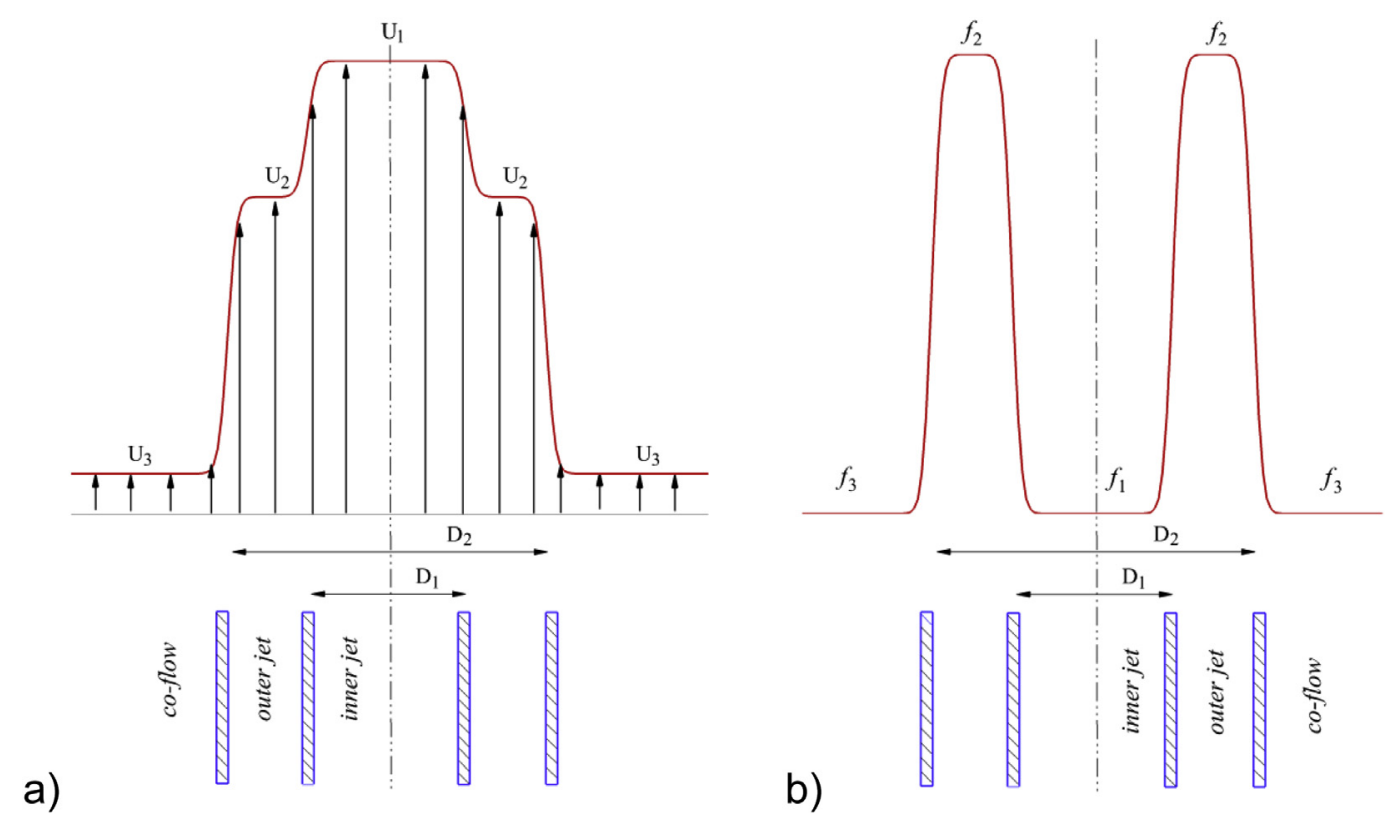

Fig. 2 - Sketch of the inlet profiles: (a) The velocity profile $U_{1}=0$ is the inner jet velocity, $U_{2}=0$ is the outer jet velocity and $U_{3}=0$ is the co-flow velocity. (b) The mixture fraction profile, we prescribe $f=1$ in the outer stream and $f=0$ elsewhere (inner stream and co-flow).

region, in order to represent the background fluctuations in natural shear flows. In general, the upstream condition is given by:

$U\left(\vec{x}_{0}, t\right)=U_{m}(r)+U_{\text {noise }}\left(\vec{x}_{0}, t\right)$

where $\vec{x}_{0}=(x=0, y, z)$ is the inlet plane, $r$ the distance from the jet axis, and $U_{m}(r)$ is the mean velocity vector which can be rewritten as comes [5]:

$U_{m}\left(\vec{x}_{0}\right)=\left\{\begin{array}{lll}\frac{U_{1}+U_{2}}{2}+\frac{U_{1}-U_{2}}{2} \tanh \left(\frac{r-R_{1}}{2 \theta_{01}}\right) & \text { for } & r \leq R_{m} \\ \frac{U_{2}+U_{3}}{2}+\frac{U_{2}-U_{3}}{2} \tanh \left(\frac{r-R_{2}}{2 \theta_{02}}\right) & \text { for } & r>R_{m}\end{array}\right.$

Where, $U_{1}$ and $U_{2}$ are the inner jet and the outer jet velocities, and $U_{3}$ is a very small co-flow, respectively. $R_{1}, R_{2}$ and $R_{m}=\left(R_{1}+R_{2}\right) / 2$, are the inner, the outer, and the averaged radii, $\theta_{01}$ and $\theta_{02}$ are the inlet momentum thicknesses of the inner and outer shear layers, respectively.

In a compressible flow, an amount of thermal energy is produced in the shear-layer region owing to molecular dissipation. Therefore, a density profile associated with the upstream velocity profile is given by Crocco-Busemann's relation.

The inlet mixture fraction profile also consists with hyperbolic tangents Fig. 2b:

$f(x=0, r)=\left\{\begin{array}{lll}\frac{f_{1}+f_{2}}{2}+\frac{f_{1}-f_{2}}{2} \tanh \left(\frac{r-R_{1}}{2 \theta_{01}}\right) & \text { for } & r \leq R_{m} \\ \frac{f_{1}}{2}+\frac{f_{2}}{2} \tanh \left(\frac{r-R_{2}}{2 \theta_{02}}\right) & \text { for } & r>R_{m}\end{array}\right.$

Note that the co-flow is always composed of species $f=0$,
The experiment carried out by Guitton et al. [29], gives an opportunity of comparing numerical simulations results and laboratory data for flows at real scales. Computation parameters were designed to fit the real coaxial jets configuration. The main differences between the various simulations come from the inlet temperature at the inner nozzle exit. All gases are assumed to be ideal. Velocity, density and pressure are non-dimensional using the values at the inner nozzle exit. Lengths are scaled on the diameter $D_{1}$ of the inner nozzle exit. The velocity ratio and radius ratio for both simulations are considered to be $r_{u}=U_{2} / U_{1}=0.7$ and $\beta=D_{2} / D_{1}=2$ respectively. Table 1 summarizes the different flow parameters studied.

The computational domain consists of a parallelepipedic box of lengths $L_{x}, L_{y}$ and $L_{z}$ where $x$ is streamwise, $y$ the transverse and $z$ the spanwise directions, respectively. The box dimensions are $15 D_{1} \times 10 D_{1} \times 10 D_{1}$.

All simulations are carried out on the same grid which consists in $500 \times 400 \times 400$ nodes, which is sufficient to resolve large scales of flow containing the wavelength of most

\section{Table 1 - Physical parameters for the air-air coaxial jet.}

\begin{tabular}{cllc} 
Flow & Parameters & $\begin{array}{c}\text { Isothermal } \\
\text { coaxial Jet }\end{array}$ & $\begin{array}{c}\text { Non-isothermal } \\
\text { coaxial jet }\end{array}$ \\
\hline Inner & Velocity $(\mathrm{m} / \mathrm{s})$ & 175 & 282 \\
jet & Temperature $\left({ }^{\circ} \mathrm{K}\right)$ & 293.15 & 792 \\
& Mach number & 0.5 & 0.5 \\
& Diameter $(\mathrm{m})$ & 0.05 & 0.05 \\
& Reynolds number & $6.25 \times 10^{5}$ & $6.25 \times 10^{5}$ \\
Annular & Velocity $(\mathrm{m} / \mathrm{s})$ & 120.5 & 197.4 \\
jet & Temperature $\left({ }^{\circ} \mathrm{K}\right)$ & 293.15 & 293.15 \\
& Mach number & 0.35 & 0.57 \\
& Diameter $(\mathrm{m})$ & 0.1 & 0.1 \\
\hline
\end{tabular}


unstable modes. The dissipation of turbulent small scales which develops in the flow from non-linear interactions is done by the PPM numerical algorithm. This dissipation is introduced by approximating the evolution of any variable across mesh through high-order interpolation followed by monotonicity constraints. The small scales are then filtered through this monotonicity which is prescribed across every mesh and every time.

\section{Results and discussion}

\section{Validation}

Fig. 3 shows the downstream evolution of the axial mean velocity compared to that done by Guitton et al. [29]. Regarding the potential core length, the figure shows that the potential cone of the inner jet is longer in comparison with that of the outer jet. We recall that potential cone is defined as $\langle u\rangle=0$. $95 U_{1}$ on the axis of the inner jet $(r=0)$ and $\langle u\rangle=0.95 U_{2}$ on the axis of the outer jet $\left(r=0.75 D_{1}\right)$. As Guitton et al. [29] considered $l_{\text {core }}^{\text {inner }} \approx 12 D_{1}$ and $l_{\text {core }}^{\text {outer }} \approx 3.5 D_{1}$, the present results reveal some deviation compared to Guitton et al. results [29] due to the initial momentum thickness taken in the present simulations.

Fig. 4 illustrates the downstream evolution of the rootmean-square (r.m.s) velocity component at the centerline of the case studied. As it can be observed from the figure, the r.m.s are in agreement with those obtained by Guitton et al. [29]. The turbulence intensity on the inner and outer axis of the jet is initially weaker up to $x / D_{1} \approx 4$, which is awhile different in terms of quality from the results acquired by Guitton et al. [29], which emphasizes taking into account more realistic initial conditions. Although the growth rate of turbulence intensity is in agreement with that predicted experimentally by Guitton et al. [29], the maximum deviation is about $14 \%$ and $17 \%$ for inner and annular jet respectively.

\section{Isothermal coaxial jet}

In this first section, we start examining the main mechanism of transition to turbulence and mixing behaviour of the isothermal coaxial jet. Additionally, this section can be regarded as a reference case for comparison with the nonisothermal coaxial jet in order to quantify the impact of gradient temperature on the flow development and turbulent mixing process.

\section{- Flow dynamics}

The flow visualizations are displayed in Fig. 5 showing the isosurfaces of the positive $Q$ (is the second invariant of the velocity-gradient tensor) for isothermal coaxial jet coloured using the value of streamwise vorticity $\omega_{x}$. Hunt et al. [42] and Dubief and Delcayre [43] introduced the concept of $Q$ criterion as a good indicator to trace the eddy structures. Therefore, up to $x / D_{1}=6$ which the first level of transition occurs, KelvinHelmholtz instability causes that outer shear layer quickly roll up into quasi-axisymmetric primary vortex rings.

It can clearly be seen from Fig. $5 \mathrm{a}$, in contrast to the outer shear layer, instabilities begin later in the inner shear layers and they are formed around $x / D_{1}=7$. The apparition of primary vortex rings is followed by the appearance of the pairs of counter-rotating streamwise vortices that emerge in the outer and inner braid region and are stretched both between two consecutive inner primary vortex rings and between two consecutive outer primary vortex rings Fig. $5 \mathrm{~b}$.

Fig. 5b shows clearly the production of intense ejections marked by the appearance of mushroom-shaped structures. These ejections phenomenon are caused by the intense streamwise vortices, which pump fluid from the outer jet core towards the co-flow by entrainment. It should be noted that the inner streamwise vortices form at a further downstream distance than the outer vortices which develop shorter downstream.

Moreover, Fig. $5 \mathrm{~b}$ also reveals that the inner primary vortex rings are trapped in the free spaces between two consecutive outer primary vortex rings. At further downstream locations, the flow quickly reaches a state of fully-developed turbulence. Fig. 6 , shows the instantaneous contours of the vorticity fields in the transverse section at several downstream locations (initial, transition and fully-developed turbulence regions). At $x / D_{1}=4.5$, it can be clearly seen that the azimuthal instabilities in the outer shear layer start quicker than in the inner shear layer. Near the exit (at $x / D_{1}=13.5$ Fig. 6), an abrupt increase in small-scale turbulence level is observed. a)

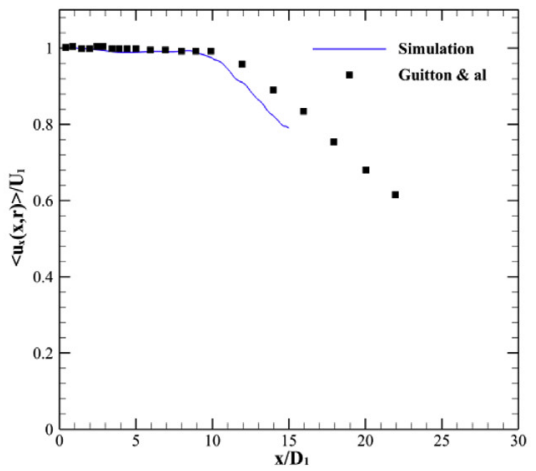

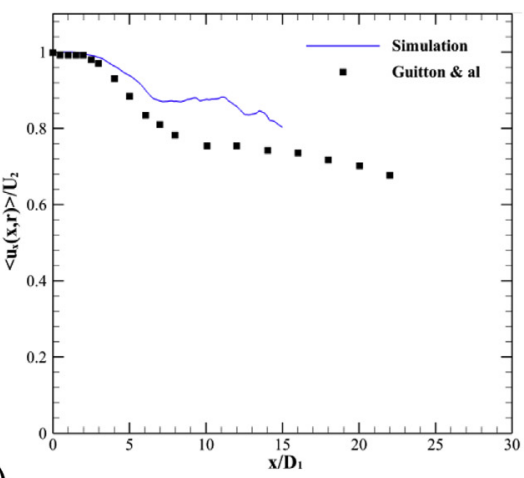

b)

inner jet $\left(r / D_{1}=0\right)$. (b) In the outer jet $\left(r / D_{1}=0.75\right)$. The numerical results are compared with the measurements by Guitton et al. [29]. 


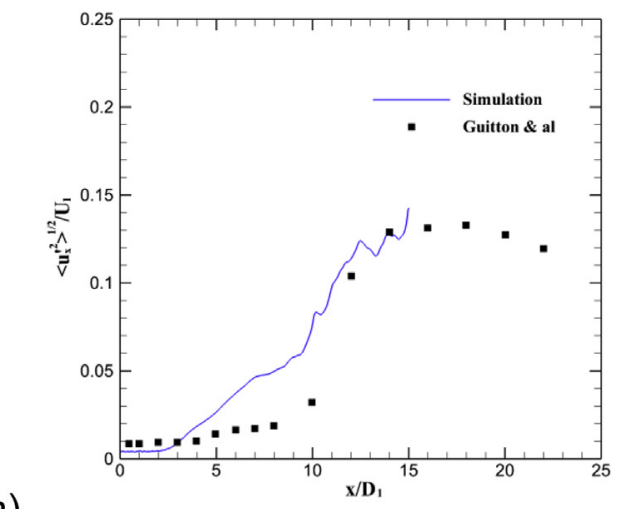

a)

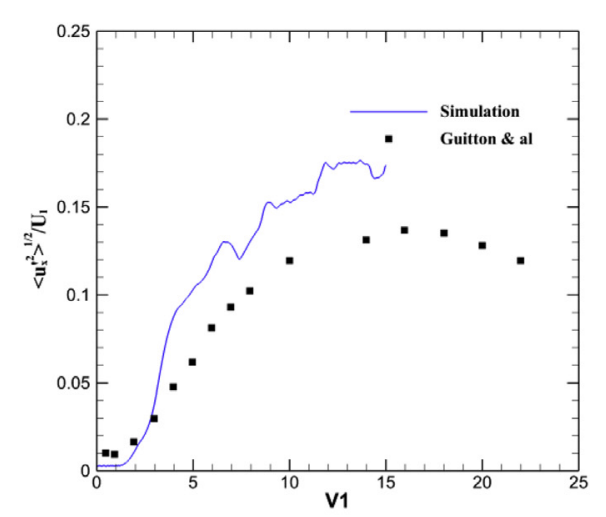

b)

Fig. 4 - r.m.s axial velocity profiles. (a) In the inner $\left(r / D_{1}=0\right)$ jet. (b) In the outer jet $\left(r / D_{1}=0.75\right)$. The numerical results are compared with the measurements by Guitton et al. [29].

\section{- Mean flow statistics}

Radial and longitudinal of mean axial velocity component and r.m.s of the velocity fluctuation are provided by Figs. 7 and 8 respectively. Using quantitative analysis, the mean and r.m.s profiles are acquired through a temporal average and an azimuthal direction of the scalar and dynamic domains.

The explicit Favre average is considered. Four flow times are adjusted as an averaging period in order to calculate mean fields and the turbulence intensities. One flow time, therefore, is determined as the time of the flow passing the computational domain.

The following relation is used to compute the turbulence intensity:

$\phi^{\prime}=\sqrt{\left\langle\varphi^{2}\right\rangle-2\langle\varphi\rangle \frac{\langle\rho \varphi\rangle}{\langle\rho\rangle}+\frac{\langle\rho \varphi\rangle^{2}}{\langle\rho\rangle}}$

where $\langle\varphi\rangle$ is the temporal average and is as comes:

$<\varphi(x, y, z)>=\frac{1}{T} \int_{t_{0}}^{t_{0}+T} \varphi(x, y, z, t) d t$ where: $\varphi(x, y, z)=U_{i=1,2,3}$ or, $t=4 L_{x} / U_{1}$ we denote mean quantities by $<$. $>$.

Fig. 7a presents the downstream evolution of the mean axial velocity in both central and annular jets. In the case studied the axial velocity is staying unchanged up to $x / D_{1} \approx 11$ at the centerline $\left(r / D_{1}=0\right)$ and $x / D_{1} \approx 3.5$ at the centre of the annular jet $\left(r / D_{1}=R_{m}\right)$. This exhibits that, there are two potential core regions, at the inner and outer jet.

Fig. $7 \mathrm{~b}$ shows the radial evolution of the mean axial velocity at several downstream locations. We can clearly see that the jet keeps a profile shape similar to the inlet one until the transition stage.

Due to radial diffuse of linear momentum which carries momentum from the outer annular jet into the co-flow and from inner to outer jets, the axial velocity after the end of each potential core is developed. Moreover, at $x / D_{1} \approx 6$ radial mean velocity profile grows more rapidly in outer shear layer which indicating clearly that a large quantity of species issued from the outer annular jet invades the co-flow, conversely at the same downstream location, the inner shear layer remains dominated by molecular diffusion and velocity profile keeps a a)

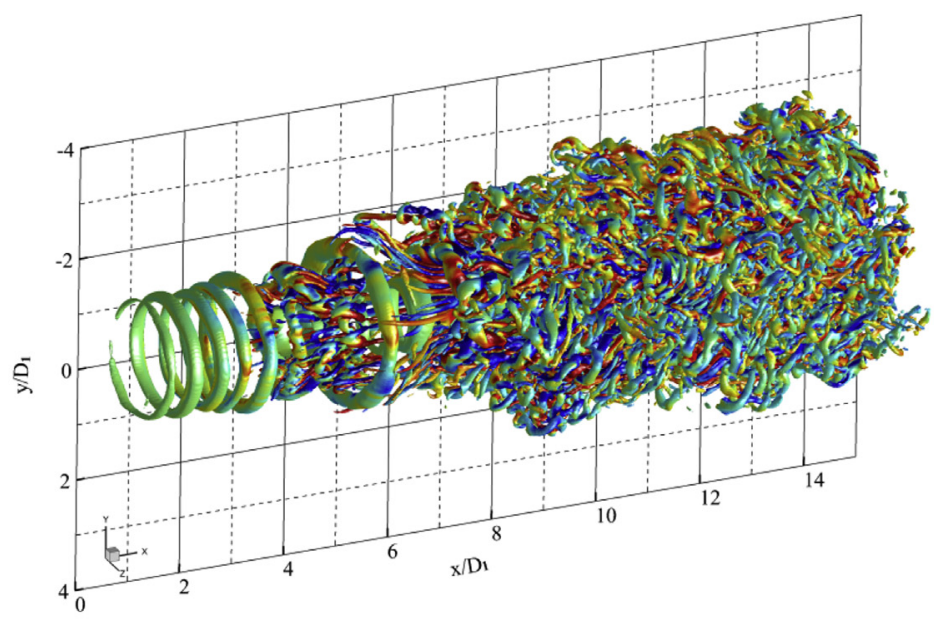

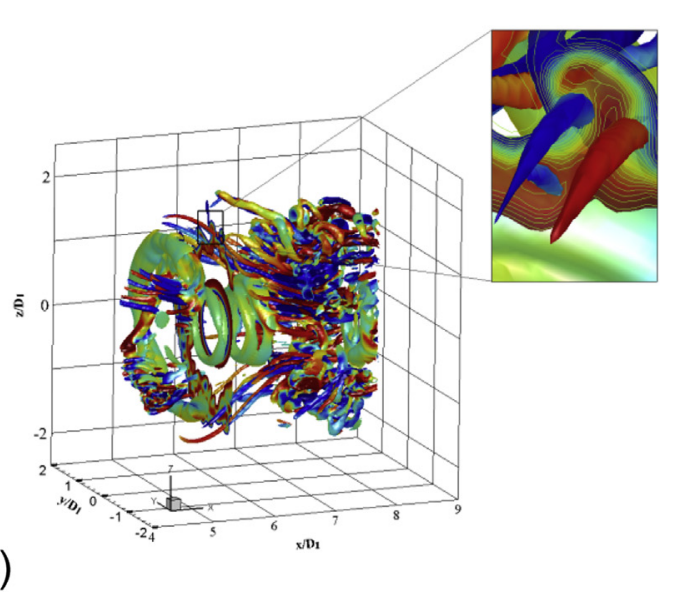

b)

Fig. 5 - (a) 3D visualization of the coherent vortices for the isothermal case by isosurfaces of positive $Q$ coloured by the streamwise vorticity $\omega_{\mathbf{x}}$. (b) Zoom of passive-scalar ejections by the counter-rotating streamwise vortices. 
$\mathrm{x} / \mathrm{D}_{1}=0$.

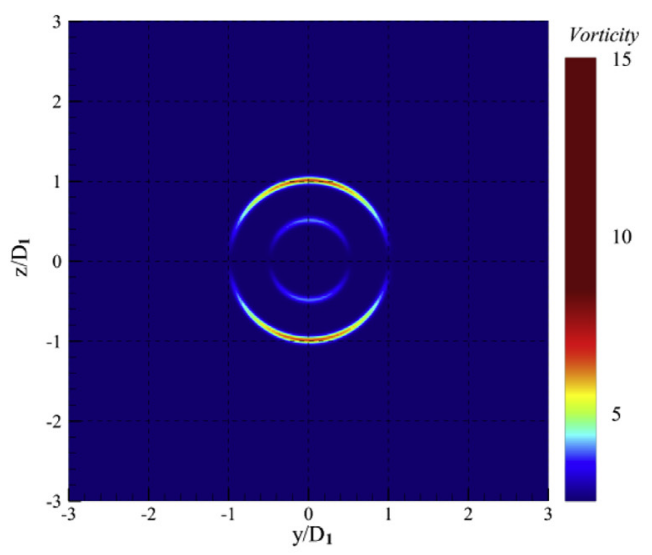

$\mathrm{x} / \mathrm{D}_{1}=4.5$

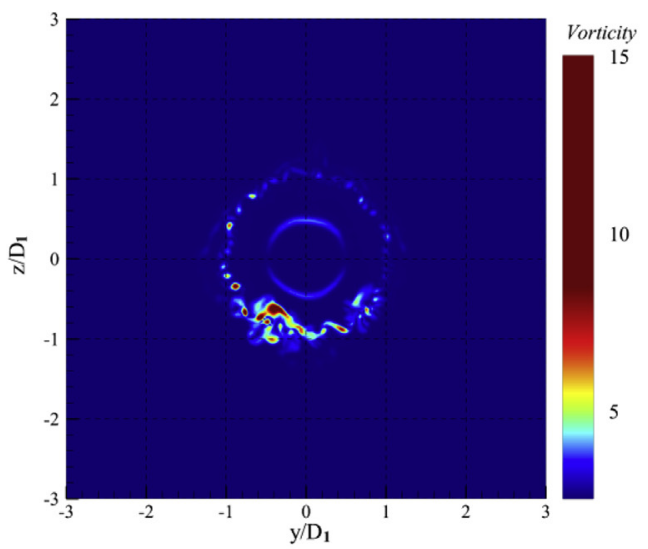

$\mathrm{x} / \mathrm{D}_{1}=7.5$

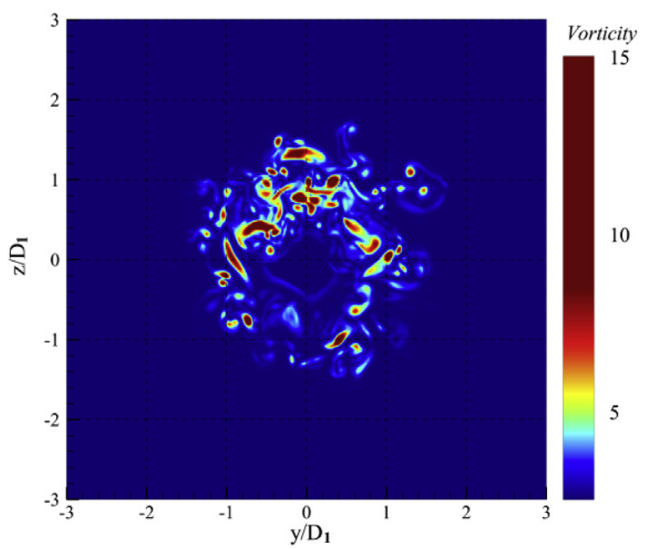

$\mathrm{x} / \mathrm{D}_{1}=3$.

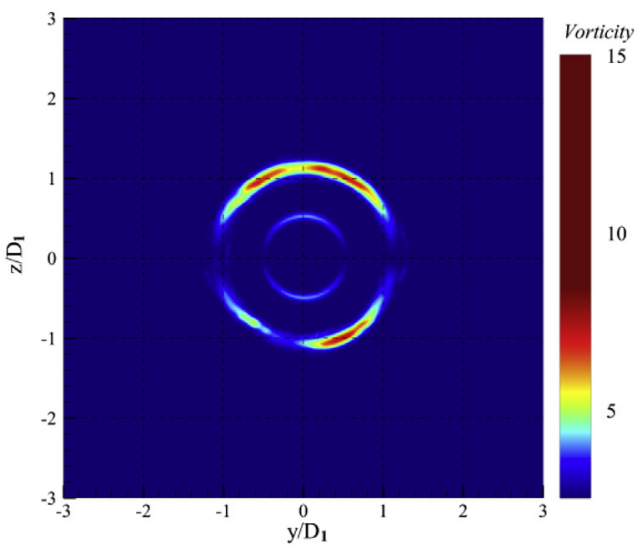

$\mathrm{x} / \mathrm{D}_{\mathbf{1}}=6$.

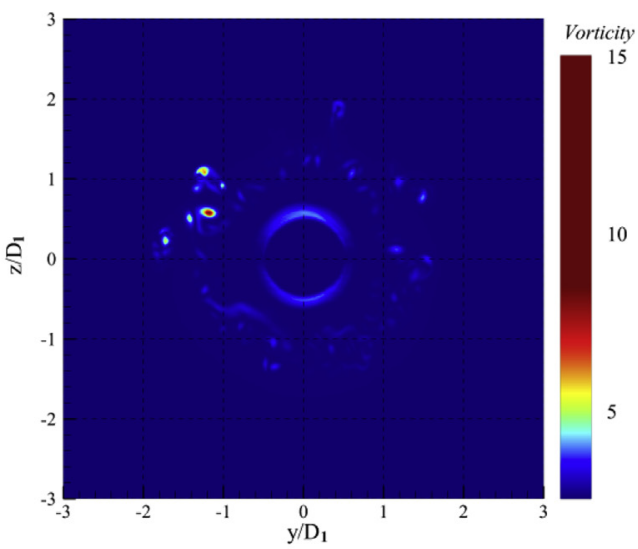

$\mathrm{x} / \mathrm{D}_{1}=13.5$.

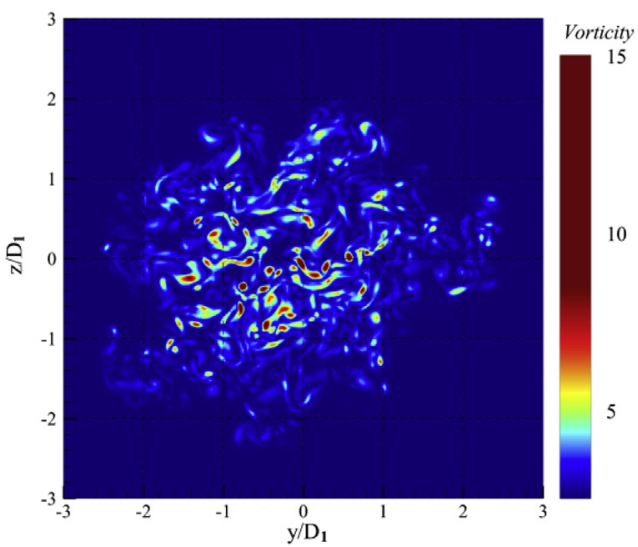

Fig. 6 - A cut through the $(z, y)$ plane of the vorticity fields.

profile similar to the inner nozzle exit. This confirms the precocious development of outer Kelvin-Helmholtz vortices.

Further downstream, for $x / D_{1}>9$ it is seen that velocity is maximized in the centerline jet $\left(r / D_{1}=0\right)$ and the velocity profile loses completely its two-layer structure. Close to the end of the domain, the radial means velocity profile is much similar to the single round jet.

Fig. 8a,b depict the radial evolution at several downstream locations and the downstream evolution of the root-mean- square (r.m.s) velocity component $\left.\left(<\mathrm{u}_{\mathrm{x}}^{\prime 2}\right\rangle^{1 / 2}(x, r)\right)$ in both central and annular jets, respectively. It can be observed that from $x / D_{1}=3$ to the end of the domain the turbulent activity is increasing significantly in both inner and outer jets. This is owing to the development of outer Kelvin-Helmholtz vortices that causes a large momentum transfer from the outer annular jet towards the inner jet and co-flow. The inner peak disappears rapidly due to the strong transfer which involves a drastic pinching of the central jet by the outer annular jet. 


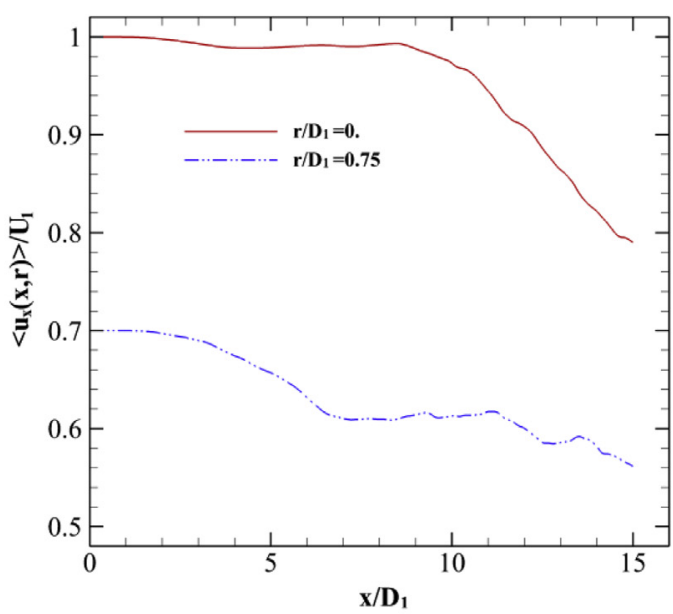

a)

Fig. 7 - Mean axial velocity component. (a) Downstream evolution in both central and annular jets. (b) Radial evolution at several downstream locations.

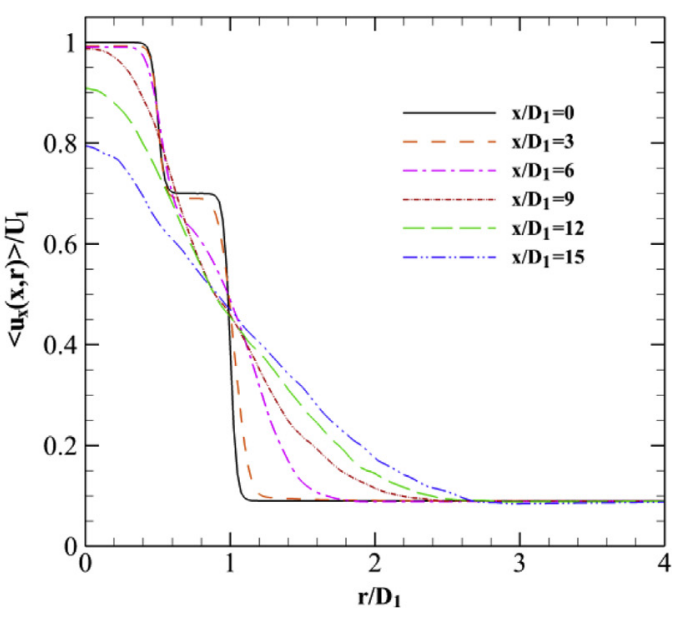

b)
At the fully-turbulent state, the maximum turbulent activity is situated in the outer annular jet. These results emphasize the domination of turbulent structures on the evolution of the flow dynamics.

The turbulent kinetic energy is employed for a better understanding of flow modification. Thus, radial kinetic energy $\left(E_{r}\right)$ and azimuthal kinetic energy $\left(E_{\theta}\right)[44,45]$ for the outer shear layer are as follows:

$E_{r}(x)=\sqrt{\frac{2 \pi}{L_{y} L_{z}} \int_{0}^{R_{m}}\left\langle u_{r}^{\prime 2}\right\rangle(x, r) r d r}$

$E_{\theta}(x)=\sqrt{\frac{2 \pi}{L_{y} L_{z}} \int_{0}^{R_{m}}\left\langle u_{\theta}^{\prime 2}\right\rangle(x, r) r d r}$

For the inner shear layer following relations are defined

$E_{r}(x)=\sqrt{\frac{2 \pi}{L_{y} L_{z}} \int_{R_{m}}^{\infty}\left\langle u_{r}^{\prime 2}\right\rangle(x, r) r d r}$

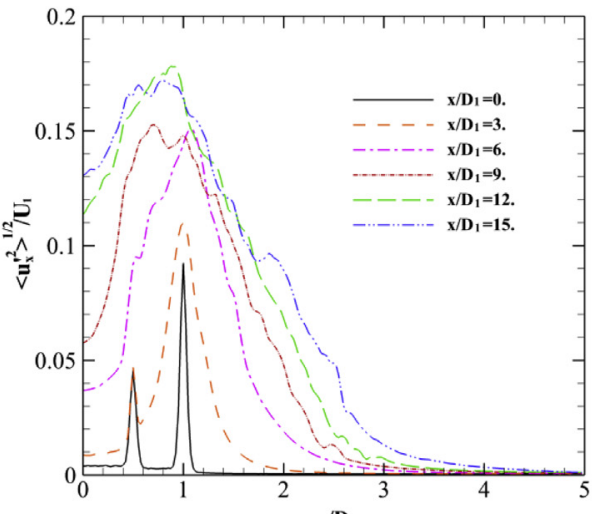

a)

$\mathbf{r} / \mathbf{D}_{1}$

b)

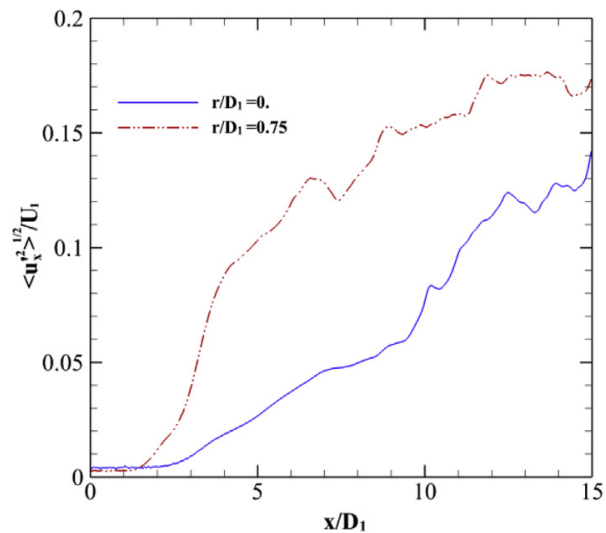

where $u_{r}^{\prime}$ and $u_{\theta}^{\prime}$ are the radial and azimuthal component of fluctuating velocity respectively. $E_{r}$ and $E_{\theta}$ are directly associated with the Kelvin-Helmholtz instabilities and azimuthal instabilities, respectively.

Fig. 9 illustrates the downstream evolution of the radial and azimuthal turbulent kinetic energy on the outer and inner jets. For $x / D_{1}<10$, it can be clearly seen that for the outer annular jet, the contribution of the radial turbulent kinetic energy is greater than that of azimuthal one; this implies that the growth of the vortex rings is largely dominating the evolution of the azimuthal instabilities. In addition, the higher contribution of azimuthal turbulent kinetic energy corresponds to the development of streamwise vortices that initiate the transition of the jet to the turbulence state. Beyond $\left(x \approx 13 D_{1}\right)$ the transition region, the contribution of both quantities becomes almost equal, which means that the jet reaches the full three-dimensionalization.

Fig. 8 - r.m.s of the longitudinal velocity fluctuations. (a) Radial evolution at several downstream locations. (b) Downstream evolution in both central and annular jets. 


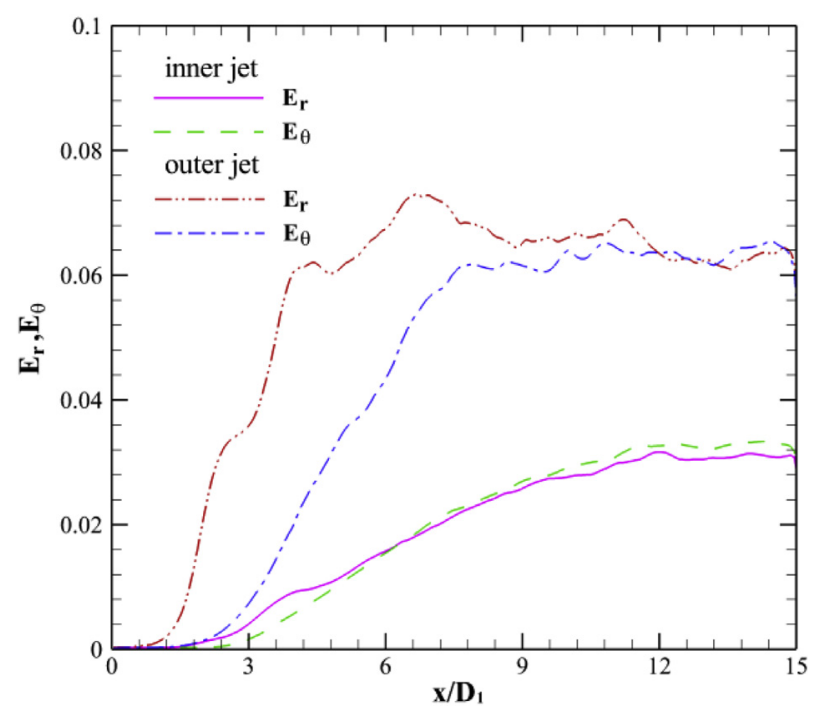

Fig. 9 - Downstream evolution of the radial and azimuthal contributions of the turbulent kinetic energy calculated for the outer and inner shear layers.

\section{Characterization of the mixing process}

Fig. 10, represents the iso-mixture fraction which is coloured by local Mach field. This iso-mixture fraction is seen as a good indicator to characterize the evolution of turbulent mixing in the coaxial jet. In addition, the characterization of the isomixture fraction plays a significant role for a better understanding of the turbulent mixing associated to turbulent combustion processes. The initial stage is characterized by the presence of two separated isosurfaces linked to the two mixing layers (inner and outer). This first stage is governed by molecular diffusion which is up to $x / D_{1}=6$ in the inner shear layer and $x / D_{1}=2$ in the outer shear layer.

After this stage, the jet is undergoing the engulfment of the two fluid streams which is a consequence of the action of amplification of Kelvin-Helmholtz vortices. After that, the inner and the outer mixing layers start to undergo a strong azimuthal instability accompanied with the emergence of the streamwise vortices which implied a strong ejection of species in mushroom-shaped structures (see Fig. 12 for $x / D_{1}=7.5$ ), this important step leads the development of highly convoluted isosurface at the fully turbulent region.

It is important to note that the inner jet is incompletely mixed with the outer jet, especially close to the end of the domain, where we can observe the presence of unmixed spots (see Fig. 10b).

In order to examine the mixing quality, Fig. 11 shows the instantaneous contours of the mixture fraction and vorticity fields in the central plane. In Fig. 11b at the near-field region, the perturbations are weak (white-noise perturbation with an amplitude of $1 \%$ ); therefore the mixing process starts with molecular diffusion. Further downstream, the turbulent mixing activity is bloomed and becomes predominant due to the appearance of Kelvin-Helmholtz vortices. Moreover, in this region, we can observe that the development of KelvinHelmholtz vortices in the outer layer begins early compared with those arising in the inner layer.
Furthermore, the turbulent mixing grows along the plane and the two jets (inner and annular) are merged at the near outflow region in order to make a single mixing zone identical to classical jets.

Fig. 12, shows a cut through the $(\mathrm{z}, \mathrm{y})$ plane of instantaneous contours of the mixture fraction fields. The figure illustrates nicely the different main stages of mixing evolution from the entrance of the domain to the exit which can be summarized as follows: initial laminar region, transition and fully-developed turbulence regions).

At $x / D_{1}=4.5$, it can be clearly seen that the turbulent mixing activity initiates early in the outer annular jet due to the appearance of the coherent vortices, in contrast, the mixing in the inner shear layer remains dominated by the molecular diffusion. Atx $/ D_{1}=7.5$, the figure illustrates clearly the ejection phenomenon marked by the mushroom-shaped structures. We recall that the formation of these structures is due to the longitudinal counter-rotating vortices (see Fig. $5 b)$. Near the end of the computational domain $x / D_{1}=13.5$ (Fig. 12), indicate an abrupt increase in small-scale turbulence level which plays the main role to improve the turbulent mixing. However, we can remark that the mixture remains incomplete in this region.

The evolution of the mean mixture fraction in the downstream direction and several sections of the jet are depicted by Fig. 13a,b respectively. It can be observed that the axial development of the mixing fraction $\langle f\rangle$ is equal to 0 and 1 for $x / D_{1}<8$ and $x / D_{1}<3$ of the inner and outer jet respectively. The radial profiles of the mean mixture fraction at the different downstream locations indicate that for values of $x / D_{1}<8$ and $x / D_{1}<3$ which are for the inner and outer jets respectively, the mixing fraction alters at the interfaces between the inner and outer jet and the ambient fluid. This variation is due to the molecular diffusion which mixes species in the radial direction. At the downstream locations, due to momentum transfer, the species enter to the central jet. Moreover, species of the annular jet diffuses into the co-flow at these downstream locations.

Fig. 13a shows that the diffusion of the species into the shear layers enhances the mixing activity following by a decrease in the annular jet and an increase in the central jet. The increase of the mixture fraction in the inner jet is less important than the decrease of the mixture fraction in the outer jet, this is due to a delay in developing Kelvin-Helmholtz vortices in the inner shear layers. This delay makes the mixture less effective at the end of the computational domain where $\langle f\rangle$ is less than 0.5 . It should be pointed out that $\langle f\rangle$ $=0.5$ is shown as a good indicator to characterize the mixing efficiency in the turbulent mixing coaxial jet.

In Fig. 14 the r.m.s mixture fraction reveals that the turbulent mixing activity in the outer jet initiates faster than in the inner jet. This is mainly due to the earlier development of KelvinHelmholtz vortices that play a major role to improve mixing.

Beyond to the $x / D_{1} \approx 4.5$, we can see clearly that outer r.m.s mixture fraction profile growth rapidly and reaches their maximum value $25 \%$ at $x / D_{1}=5$ in this downstream location the turbulent mixing becomes more and more significant. This is because of counter-rotating streamwise vortices (see Fig. 5b), which allow the ejection of species drastically from the outer core towards the co-flow and initiates the three- 


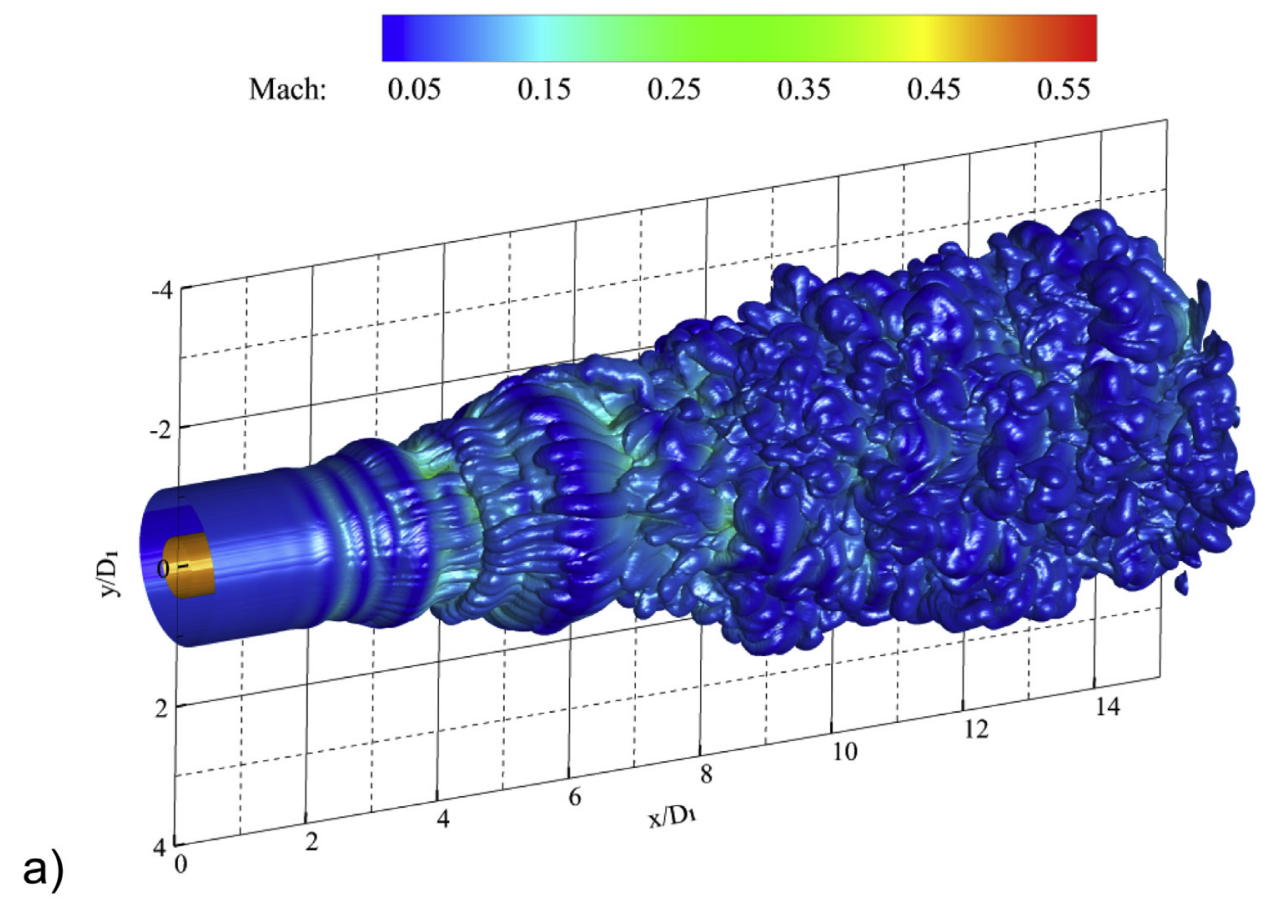

a)

b)

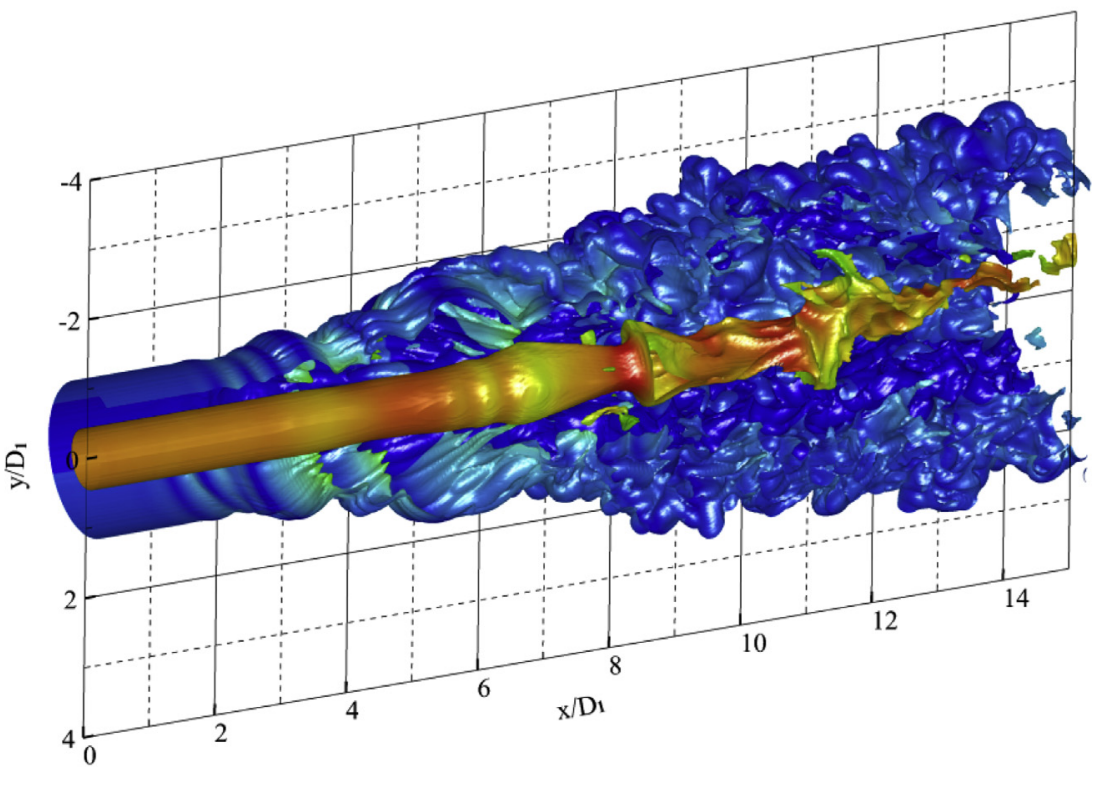

Fig. 10 - Global view of the natural isothermal coaxial jet case: (a) isosurface of mixture-fraction. (b) Cut view of $f=0.15$ mixture-fraction isosurface coloured by the local Mach number.

dimensionality of the jet. Until $x / D_{1} \approx 6$, the radial profiles at various axial positions Fig. 14b indicate two distinct peaks situated in the inner and outer shear layers, respectively. These two peaks represent the imprints marked by the inner and outer large structures, respectively.

The turbulent mixing activity is confined in narrow thickness $r / D_{1}<2$ in the transition region, however, it extends downstream to reach the value $\left(r / D_{1}=3.3\right)$ at the end of computational domain as shown in the Fig. 14b. Moreover, it is worth noting that the outer peak skewed to the right side of the jet $\left(r / D_{1}=2.7\right.$ instead of the 1$)$ due to the higher momentum transfer between the outer annular jet and the ambient.
Near to the exit domain $\left(x / D_{1}=15\right)$, large amounts of fluid issued from outer annular jet invade the co-flow and the inner jets that allow the merging of the two mixing layers and the formation of a single mixing zone similar to that obtained in the single jet.

To examine the evolution and the characterization of the turbulent mixing in this configuration (isothermal coaxial jet), the variations of the probability density functions which were presented and discussed by Stanley et al. [46] are performed.

Fig. 15 highlights the radial probability density functions 'PDFs' at three streamwise locations this allows to cover all main regions in the flow (initial, transition fully turbulent stages). At the upstream position of $x / D_{1}=3$ (Fig. 15a), the 
a)

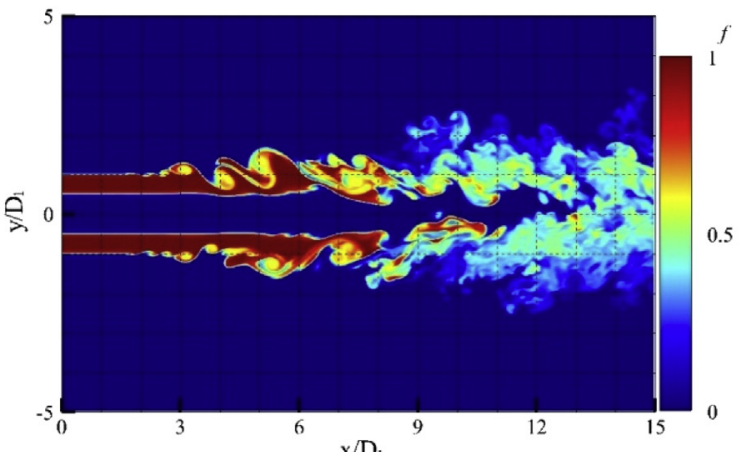

b)

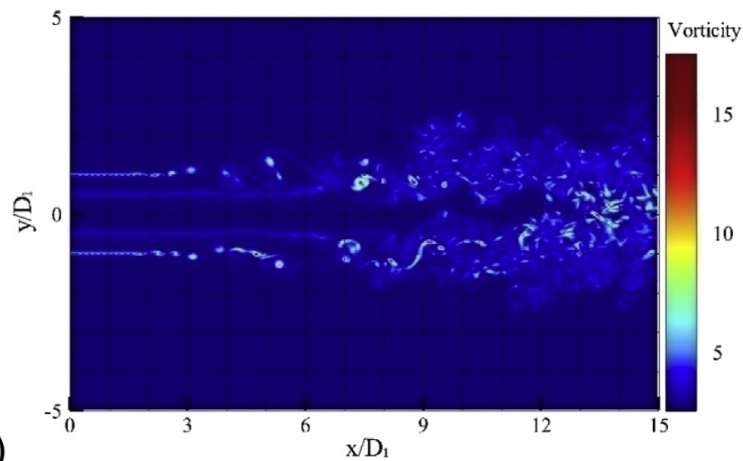

$\mathrm{x} / \mathrm{D}_{1}$

Fig. 11 - Contours for the natural isothermal jet in the central plane. (a) mixture-fraction. (b) vorticity modulus.

shape of the PDF evidently proves that the mixing behaviour is very solely and remains confined in the inner and outer shear layers, respectively, due to the molecular diffusion. As it was already discussed up to $x / D_{1}=6$ the turbulent mixing has not yet started to develop in the flow.

Further downstream at $x / D_{1}=11.5$, Fig. $15 \mathrm{~b}$ reveals a range in which the mixing fraction at each radial position for $r / D_{1}>$ 0.5 is likely to occur between the outer jet and the ambient. This is directly associated to the earlier development of the counter-rotating streamwise vortices which pump mixture from the outer annular core towards the co-flow. This leads to improve the turbulent mixing process and the growth of mixture envelope in this region. At the same downstream location at $x / D_{1}=6$, for $r / D_{1}>0.5$ it can be seen that the value of the mixing fraction remains rather much weak but nonnegligible. This explains the delay of developing KelvinHelmholtz vortices in the inner jet which play a major role in the mixing process in this region.

Close to the exit, Fig. 15c shows that the inner and the outer mixing layers are merged into a single-mixing layer. A large range in which the mixing fraction at each radial position for $\left|r / D_{1}\right|<2.2$ is likely to occur between the outer jet and the ambient.

Moreover, a further examination reveals a trace of unmixed species with the value $f \approx 1$ is detectable in the inner jet. This confirms that the outer jet has not well penetrated the jet center which means also that the mixing between annular and inner jet is not quite homogeneously distributed inside the center jet.

\section{Non-isothermal coaxial jet}

\section{- Flow dynamics and statistics}

This section examines the details of the impact of temperature gradients on flow dynamics evolution and mixing enhancement. The findings of the global flow structure and statistics are compared to the reference case (isothermal coaxial jet). It seems worth recalling that both cases have been carried out under the same inlet conditions. The only difference between the coaxial jets occurs in the temperature of the inner jet. Indeed, the temperature ratio between the annular jet and the co-flow is $T_{2} / T_{3}=1$. for both configurations, whereas the non-isothermal configuration has an inner jet temperature $T_{1}=2.7 T_{3}$. The flow parameters of the hot coaxial jets are summarized in Table 1 .

A global overview of the flow dynamics evolution of nonisothermal configuration is displayed in Fig. 16, which represents the visualization of positive $Q$ isosurfaces coloured by the streamwise vorticity $\omega_{\mathrm{x}}$. We can clearly see that the scenario of the flow dynamics evolution, in this case, is much similar to that of the isothermal coaxial jet (section Isothermal coaxial jet). As pointed out earlier, we can distinguish three regions of flow development: Laminar region (1) where we see the evolution and growth of unstable modes which lead to the formation of inner and outer vortex rings in both inner and outer shear layers due to Kelvin-Helmholtz instabilities. Transition region (2) characterized by the appearance of counter-rotating streamwise vortices. The third region (3) shows the structures in the fully developed state.

The initial stage which controlled by molecular diffusion processes seems shorter in the non-isothermal case than the isothermal in the inner jet as shown in Fig. 18. Indeed, a closer look at Fig. 18 shows also that the amplification of the inner Kelvin-Helmholtz vortices (that keep their coherence until the end of the jet potential core) starts earlier in this case. The appearance precocious of Kelvin-Helmholtz vortices allows a rapidly accelerating towards to fully-turbulence stage.

Close examination of the results displayed in Fig. 17, illustrates nicely that the modifications of the transition due to the gradient of temperature (coaxial jet with heated inner jet) have a significant influence on the mean flow behaviour. Thus, in the initial stage where the potentials cores take place, we can clearly see that there is a remarkable decreasing of the inner potential core length in the non-isothermal jet as described in Fig. 17a.

It is very important to know the parameters that control the length of the inner/outer potential cores to optimize the operation of rocket engines, as well as combustion chamber design.

As pointed out by Lau [47] and more recently by Bodony and Lele [48], the potential core length in the simple jet decreases generally when the jet-exit temperature is increasing. Therefore, in the non-isothermal case, the temperature gradient between the outer and the inner jets inducing a precocious development of intense inner Kelvin-Helmholtz vortices. Hence, the streamwise vortices emergence are generated earlier leading to strong momentum and mass 
$\mathrm{x} / \mathrm{D}_{1}=0$.

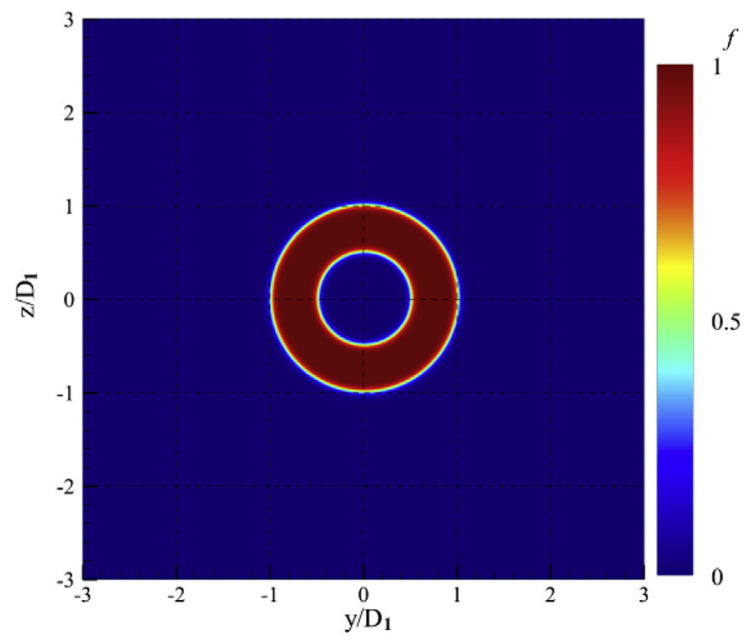

$\mathrm{x} / \mathrm{D}_{1}=4.5$

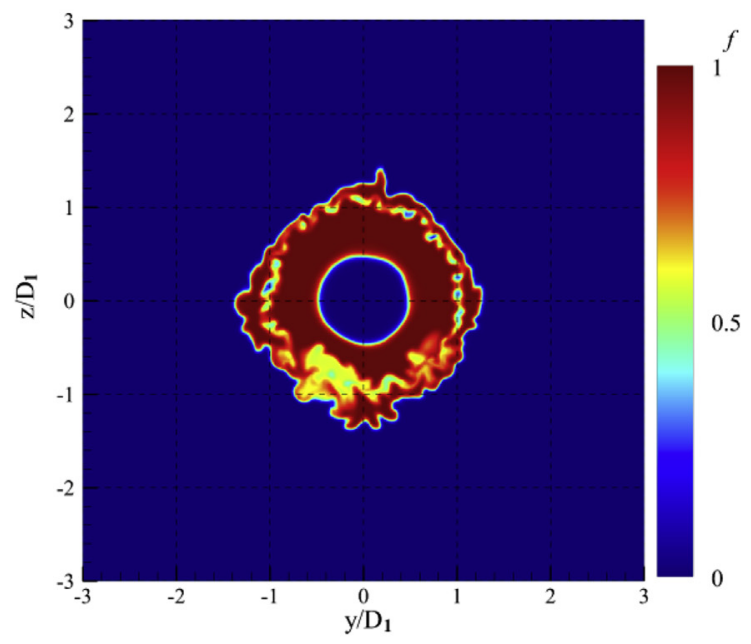

$\mathrm{x} / \mathrm{D}_{1}=7.5$

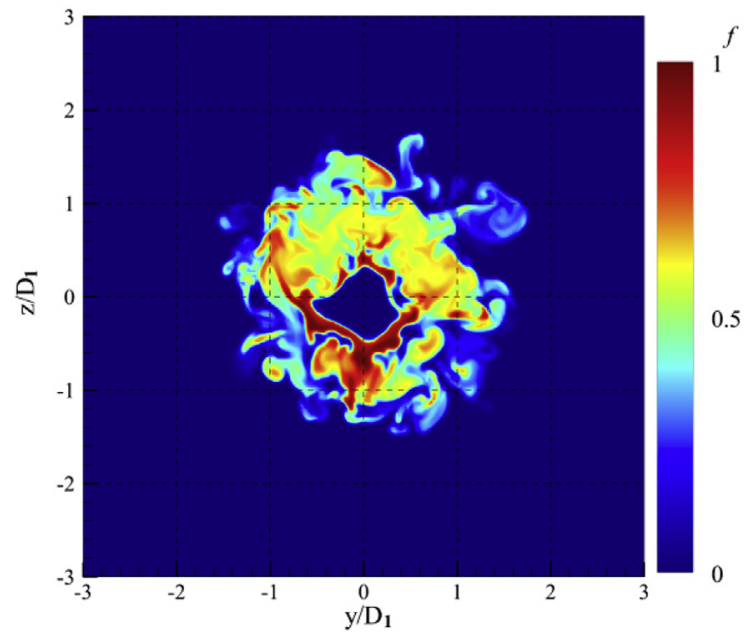

$\mathrm{x} / \mathrm{D}_{1}=3$.

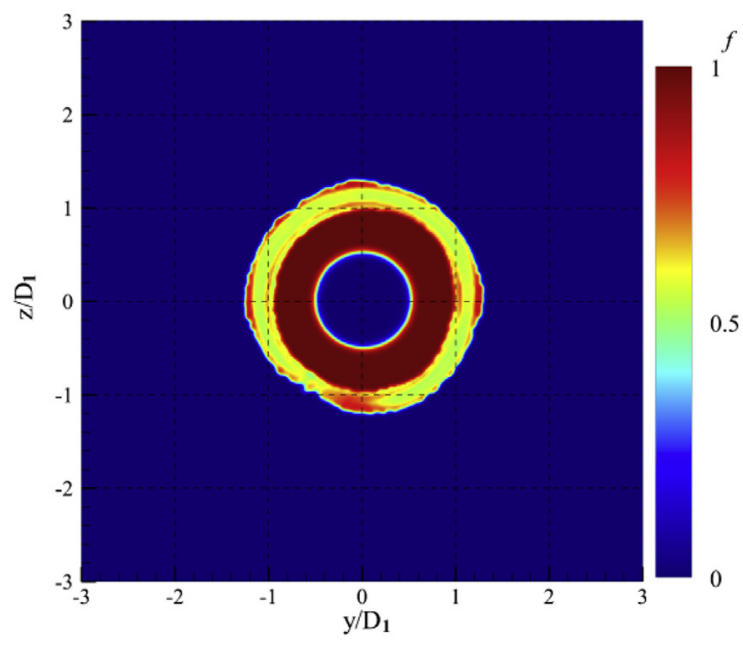

$\mathrm{x} / \mathrm{D}_{1}=6$.

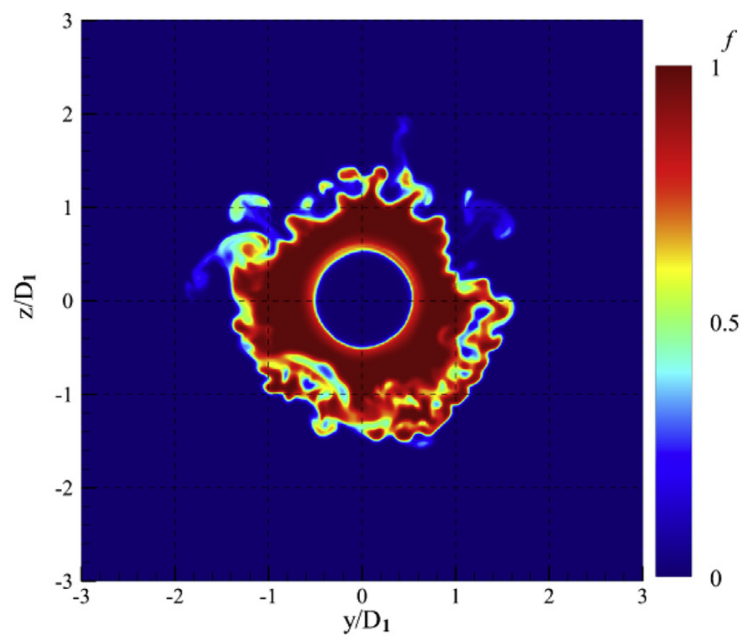

$\mathrm{x} / \mathrm{D}_{1}=13.5$.

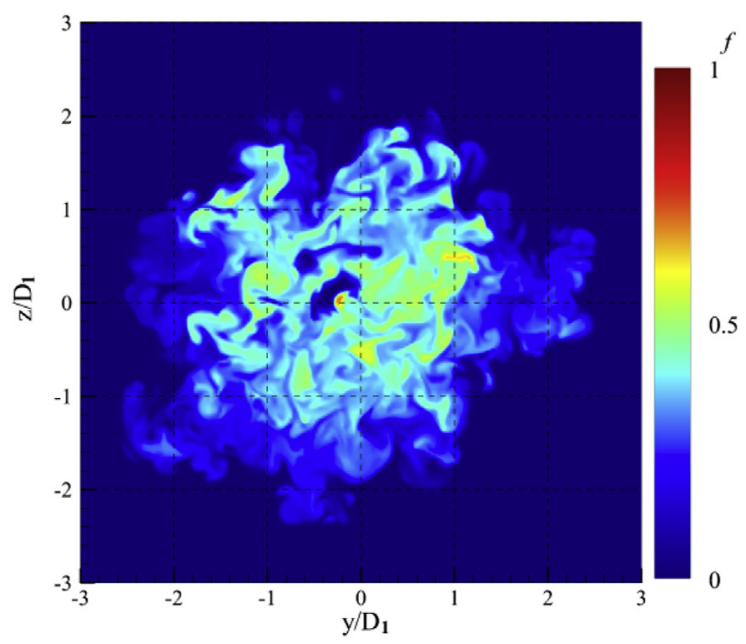

Fig. 12 - A cut through the $(z, y)$ plane of the mixture-fraction fields. 


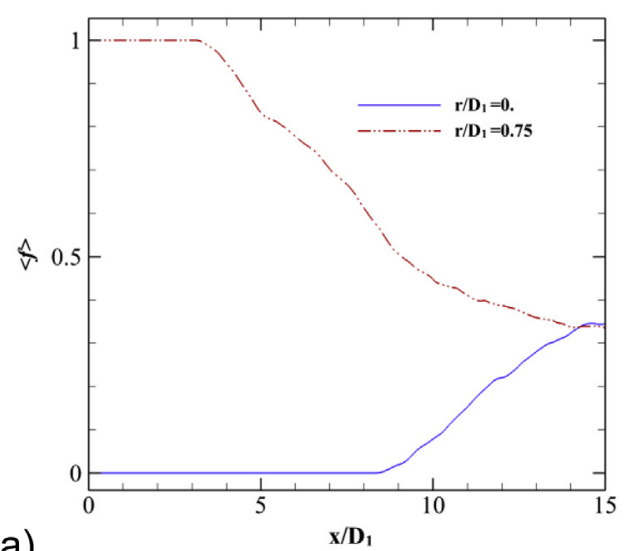

a)

Fig. 13 - Mean mixture-fraction. (a) Downstream evolution in both central (continuous line) and annular jets (dash dot dot line). (b) Radial evolution at several downstream locations.

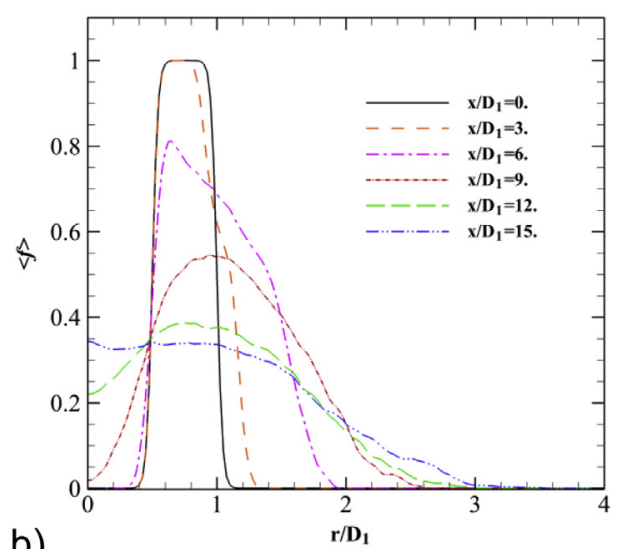

b) transfer between the outer and the inner jets accompanied by a diminution of the mean axial velocity of the inner jet (Fig. 18). As a result, the inner potential core length reduces. Conversely, the outer potential cores length in both cases remains identical as the temperature gradient between the outer jet and the co-flow is null. Additionally, the centerline distributions of r.m.s mixture fraction (Fig. 24) show that the turbulent mixing activity in the inner jet starts quicker in the non-isothermal case than in the isothermal. In agreement with previous observations and results obtained, this figure confirms that the inner potential core length is shorter in the non-isothermal jet.

Downstream of the potential core, the mean axial velocity has significantly decreased along the jet centerline which leads to homogenize the velocity at the fully-turbulence region. This is caused by the earlier intense inner KelvinHelmholtz vortices that activate the momentum transfer from the inner jet towards the annular as shown by Fig. 19 (which displays a zoom on an isosurface of $Q>0$ ).

Moreover, Fig. 17b illustrates that at the end of computational domain $\left(x / D_{1}=15\right)$ the velocity in the jet center is weaker in the non-isothermal case (with a maximum value of the order of $0.6 U_{1}$ ) than in the isothermal case (with a maximum value of the order of $0.8 U_{1}$ ), but the spreading of the coaxial jets is almost alike and reaches the co-flow value for $r / D_{1}=2.3$ in both cases (isothermal and non-isothermal). This shows that the flow spreading is not significantly affected by temperature gradients. The distributions of the longitudinal velocity fluctuations at several downstream locations are depicted in Fig. 20. It can be seen that the velocity fluctuations are confined in narrow thickness $r / D_{1}<2$ in the transition region, however, it extends downstream over twice.

In addition, at the end of the computational domain, the r.m.s profile shape loses completely its double peaks structure (the double peaks are the imprints of the large structures) and turns into one peak where the fluctuation reaches the maximum value of $14.5 \%$ at the centerline jet, conversely to the isothermal case where the maximum of intense turbulent activity is localized in the annular jet Fig. 8 a.

In this region, the large-scale eddy structures break down, which causes an abrupt increase in the small-scale turbulence level and the flow quickly enters the fully developed turbulent state. a)

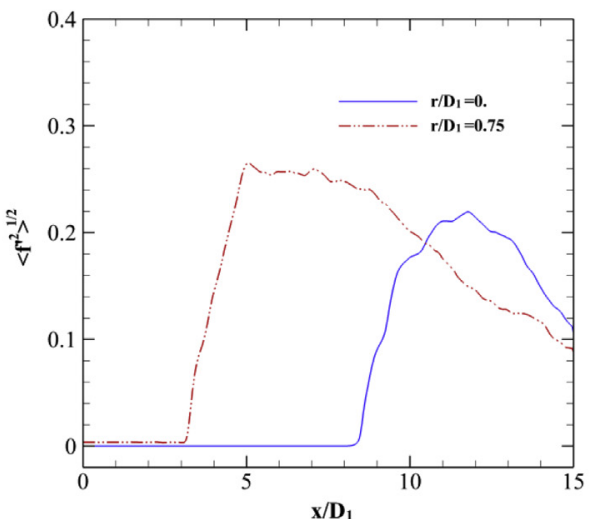

Fig. 14 - r.m.s of the mixture-fraction. (a) Downstream evolution in both inner (continuous line) and annular jets (dash dot dot line). (b) Radial evolution at several downstream locations. 


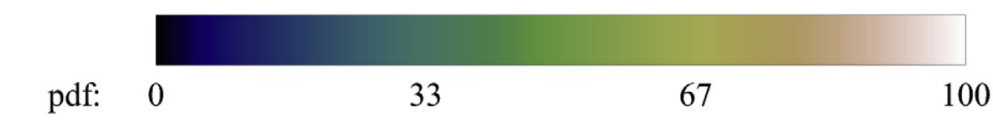

a)
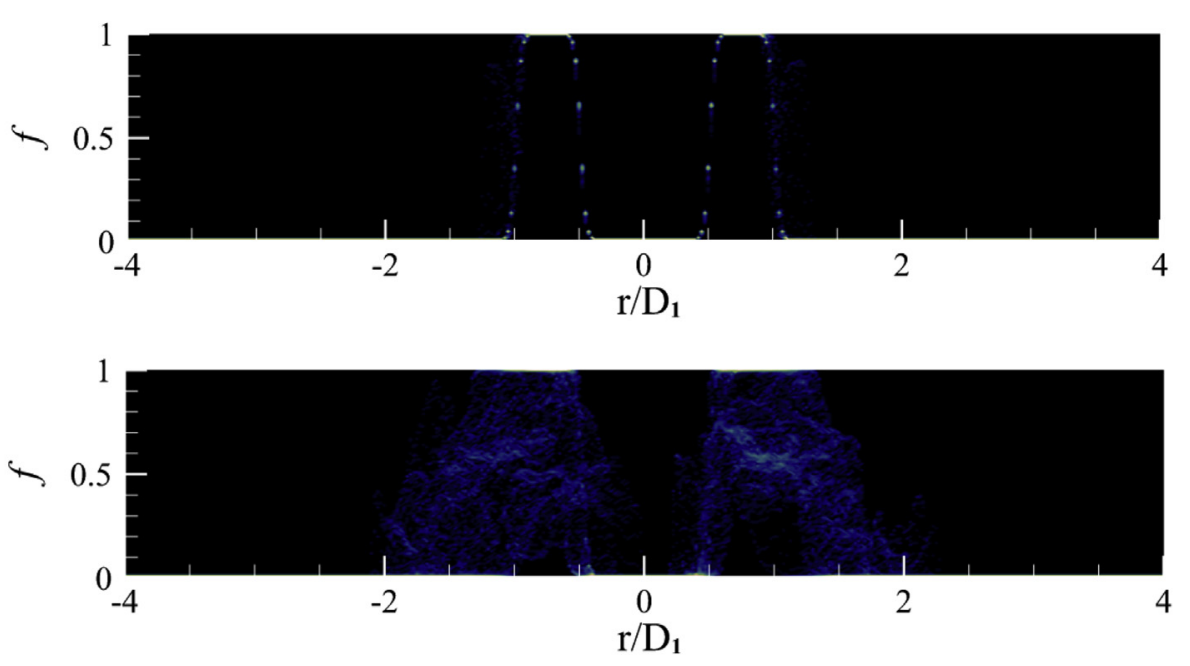

b)

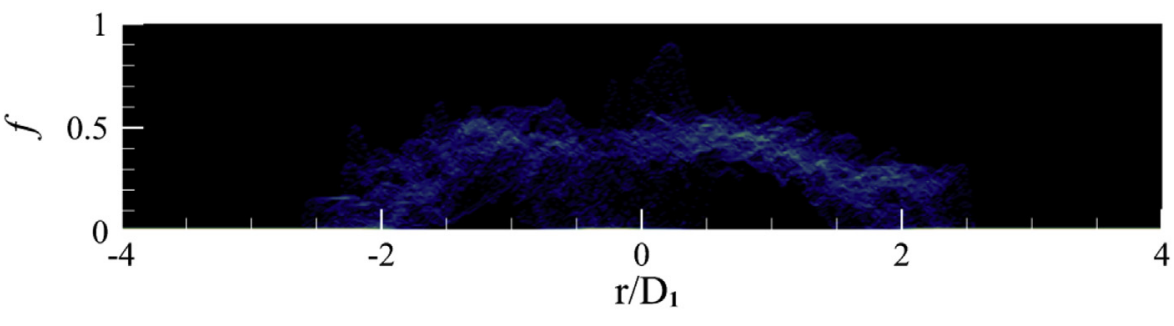

Fig. 15 - Variation of the probability density function of the mixture-fraction (PDF) for the isothermal jet at: (a) $x / D_{1}=2$. (b) $\left.x / D_{1}=11.5 \mathrm{c}\right) \times / D_{1}=15$.

Fig. 21a shows the streamwise mean velocity profiles at several downstream locations obtained for the nonisothermal case. It can be seen clearly that the profiles are qualitatively corroborating by the good agreement compared with experimental data of classical incompressible jets [49]. We have also determined the Reynolds stresses. Fig. 21(b)-(d) display the profiles of the streamwise normal, streamwise radial and tangential components of Reynolds stress tensor, respectively. From the collapse of the profiles from the current results, it can be seen that the Reynolds stress components are close to self-similarity near the outflow of the domain.

\section{- Characterization of the mixing process}

To examine the effect of temperature gradient on the mixing features, Fig. 22a shows visualizations of instantaneous contours of the mixture fraction. The mixing process at the initial stage is primarily controlled by molecular diffusion. In the inner heated jet, this stage is shorter as compared with the isothermal jet. Indeed, in the inner jet, the molecular diffusion of the laminar layer is limited to $x / D_{1} \approx 3$ instead of $x / D_{1}<6$ in the isothermal case.

The turbulent mixing activity then begins quicker due to the earlier development of coherent vortices. Finally, at the end of the computational domain $\left(x / D_{1}>10\right)$, turbulent small scales allow an intense turbulent mixing as shown by Fig. $22 \mathrm{~b}$.

The isosurface representation of the mixture fraction is coloured by local Mach field which allows a more accurate examination of the mixing evolution as depicted in Fig. 23. The initial stage is characterized by two decorrelated isosurfaces corresponding to the two mixing layers (inner and outer). It can be observed that the inner diffusion of the laminar layer is shorter in this case $\left(x / D_{1}=3\right.$ instead of $x / D_{1}=6$ in the isothermal coaxial jet). This stage is followed by the engulfment of the two fluid streams caused by the action of amplification of the Kelvin-Helmholtz vortices, which begin to be visible at $x / D_{1}=3$.

In the upstream location, the inner and the outer mixing layers start to undergo an azimuthal instability accompanied by the emergence of the streamwise vortices which eject species in mushroom-shaped structures (see Fig. 22), this is leading to a highly complex isosurface shape at a fully turbulent region. It is important to note that the outer annular jet invades deeply into the centre of the jet, which allows the increase of mixing in this configuration in contrast to the isothermal case. Moreover, the outer annular jet is completely mixed with the inner jet, especially in the vicinity of the end of the domain (see Fig. 23b). This significant result is 


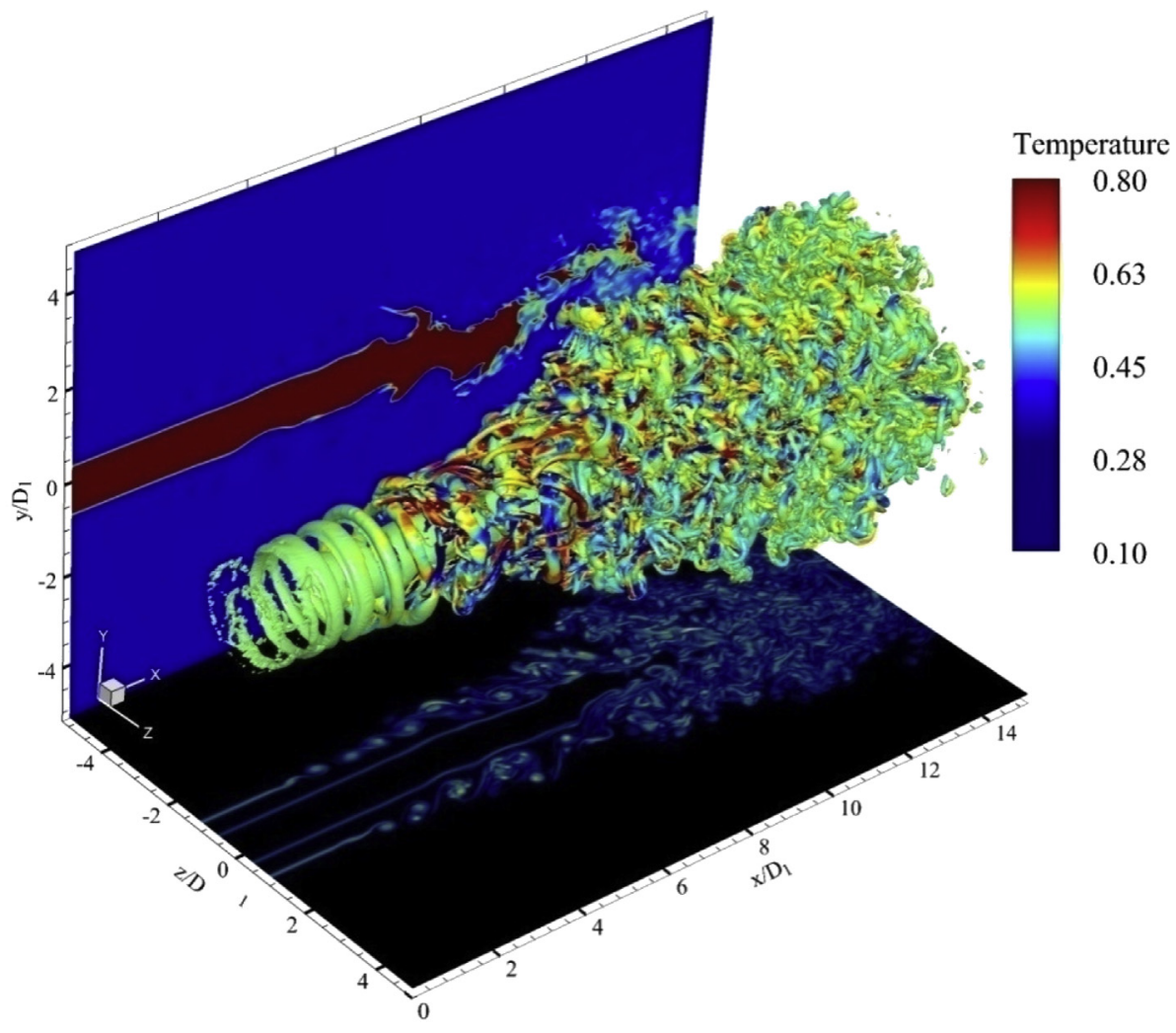

Fig. 16 - 3D visualization of the coherent vortices for the non-isothermal case by isosurfaces of positive $Q$ coloured by the streamwise vorticity $\omega_{\mathbf{x}}$ superimposed with vorticity module field (bottom side) and temperature field (back side).

undoubtedly due to the temperature gradient, which helps the enhancement of entrainment and mixing process.

Conversely to the isothermal case, further downstream of $x / D_{1}>7$, we can see that the part of the fluid issued from the outer jet which invades the inner jet is much important as compared with the isothermal case. This is due to the precocious of pairs of streamwise vortices generation on the inner shear layer which emerge between two consecutive inner Kelvin-Helmholtz vortices.

To assess the difference between the two cases, it is of interest to examine the mean and r.m.s quantities. Fig. 24 shows a comparison between the mean mixture fraction statistics for the non-isothermal and the isothermal cases. This figure illustrates that the non-isothermal coaxial jet

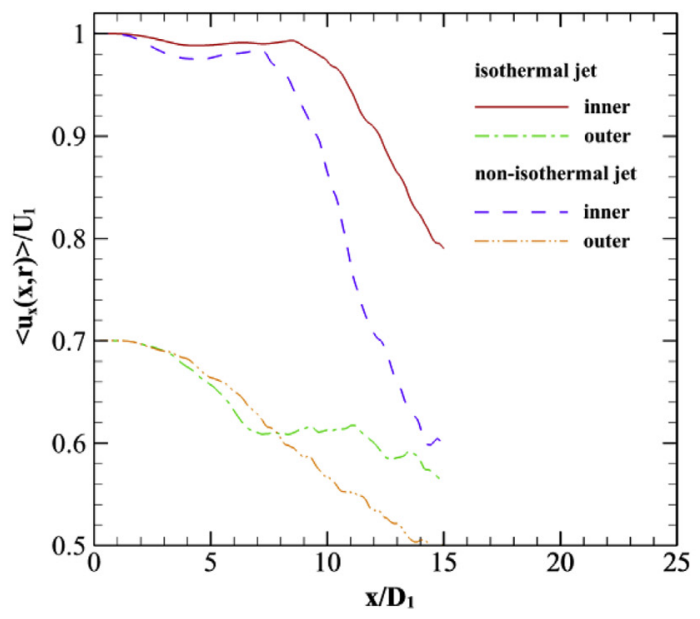

a)

b)

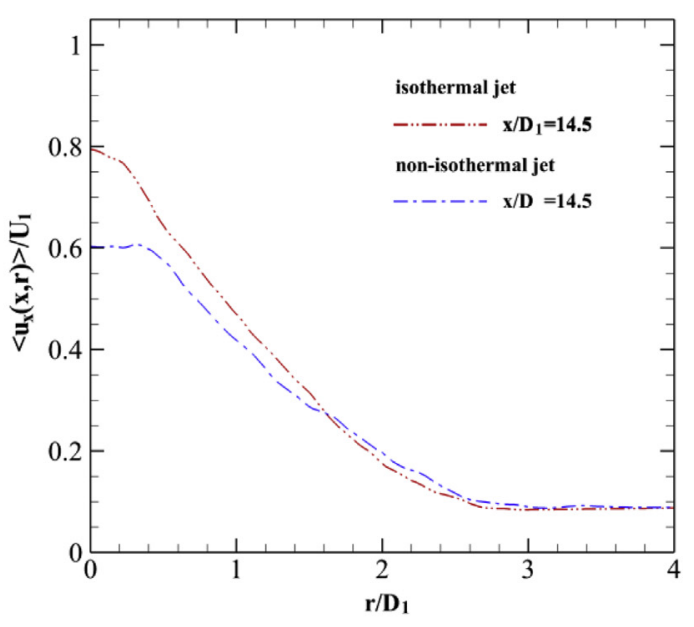

Fig. 17 - Comparison of the mean axial velocity between the isothermal and non-isothermal cases: (a) downstream evolution in the inner $\left(r / D_{1}=0\right)$ and outer $\left(r / D_{1}=0.75\right)$ jets and $(b)$ profiles at the downstream location $\left(x / D_{1}=15\right)$. 


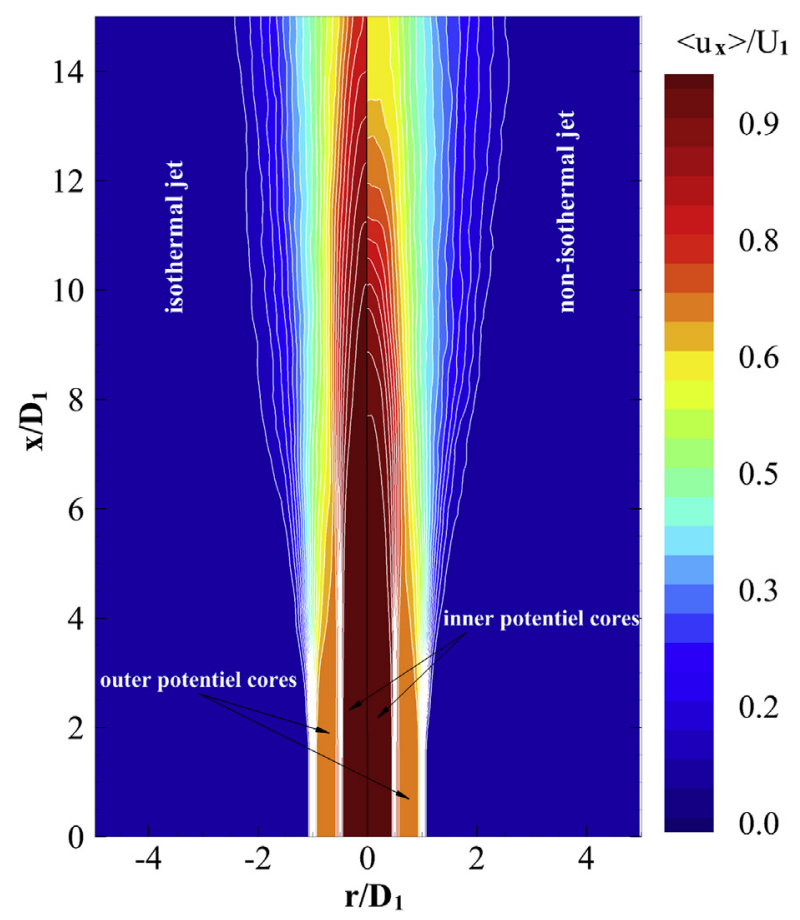

Fig. 18 - Instantaneous contours of the mean axial velocity field superimposed with contour lines to show clearly the potential cores.

starts the mixing process more upstream in the jet centre than the isothermal coaxial jet. Moreover, At $x / D_{1}=6$, the radial means mixture fraction profiles reveal that there is a strong invasion of the species issued from the outer annular jets into the co-flow in both cases, while in the inner jets the mean mixture fraction remains equal to 0 at the same downstream position. This confirms the quick development of the outer Kelvin-Helmholtz vortices. Moreover, upon closer examination, for $x / D_{1}>6$, on the jet centerline, it turns out that the mean mixture fraction in the non-isothermal jet starts to increase faster than the isothermal jet. This upshot is essentially due to the temperature gradient that induces an earlier

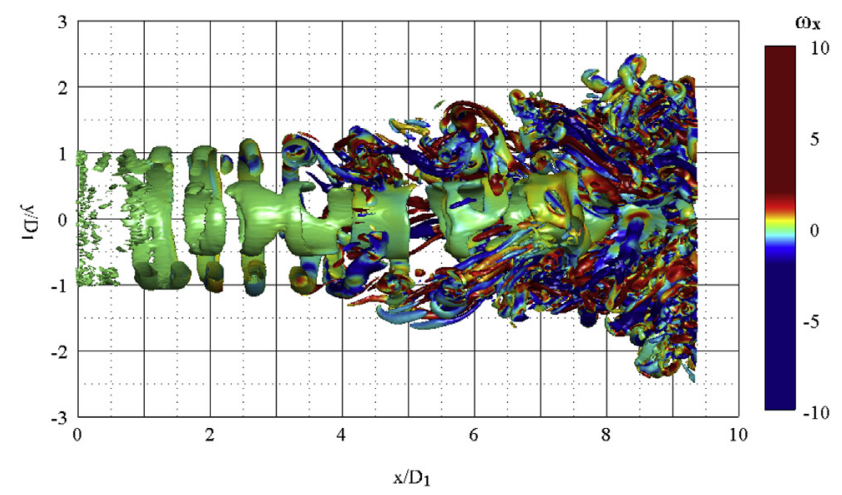

Fig. 19 - Zoom on the inner coherent structures for the non-isothermal jet. Cut view of positive $Q$ coloured by the streamwise vorticity. Large parts of the isosurfaces have been cut to see inside the flow.

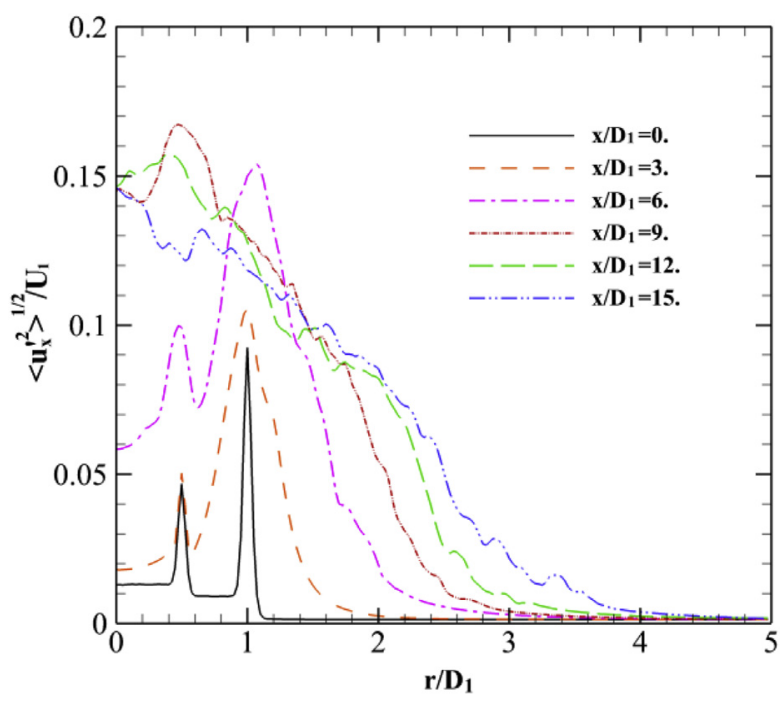

Fig. 20 - r.m.s axial velocity profiles at several downstream locations.

development of the inner Kelvin-Helmholtz vortices which help the mixing between the inner and outer annular jets.

Moreover, at $x / D_{1}=9$, the influence of the outer streamwise vortices ejections (Fig. 23a) is clearly visible through an important widening of the mean mixture fraction profile towards the ambient fluid as displayed in Fig. $24 \mathrm{~b}$.

It should be also mentioned, that the radial spreading of the mixture is almost alike and reaches the co-flow value for $r / D_{1}=3$ in both cases as can be seen in Fig. $24 b$. Close to the exit, the difference in mixing becomes more evident in the non-isothermal coaxial jet.

In order to get a better understanding of the effect of the temperature gradient on the mixture, the comparison of r.m.s mixture fraction between the non-isothermal and the isothermal is depicted in Fig. 25. These statistic quantities help to explain the previous results. The r.m.s mixture fraction $\left\langle f^{\prime 2}\right\rangle^{1 / 2}$ starts to increase dramatically at $x / D_{1} \approx 6.5$ in the non-isothermal case, instead of $x / D_{1} \approx 9$ for the isothermal case. This earlier increase can be associated with the strong mixing activity caused by the earlier development of KelvinHelmholtz vortices that play a major role to increase the turbulent intensity.

Moreover, in the inner jet, the precocious increases of mixture fraction fluctuations in the non-isothermal case at $\left(x / D_{1} \approx 6\right.$.5instead of $\left.x / D_{1} \approx 9\right)$ implying a faster turbulent mixing in this region.

Note that beyond $x / D_{1}=11.5$, the r.m.s quantities are smaller in the non-isothermal case, showing a diminution of the turbulent mixing activity linked to a better species homogenization.

It should be noted that in the outer jet the r.m.s mixture fraction is not affected by the temperature gradient. This result justifies the identical radial spreading of both coaxial jets (non-isothermal and isothermal) previously founds (Fig. 24b).

A further examination of the mixing of the non-isothermal configuration is based on the radial probability density functions PDFs at the transition and in the fully-turbulent stages. 

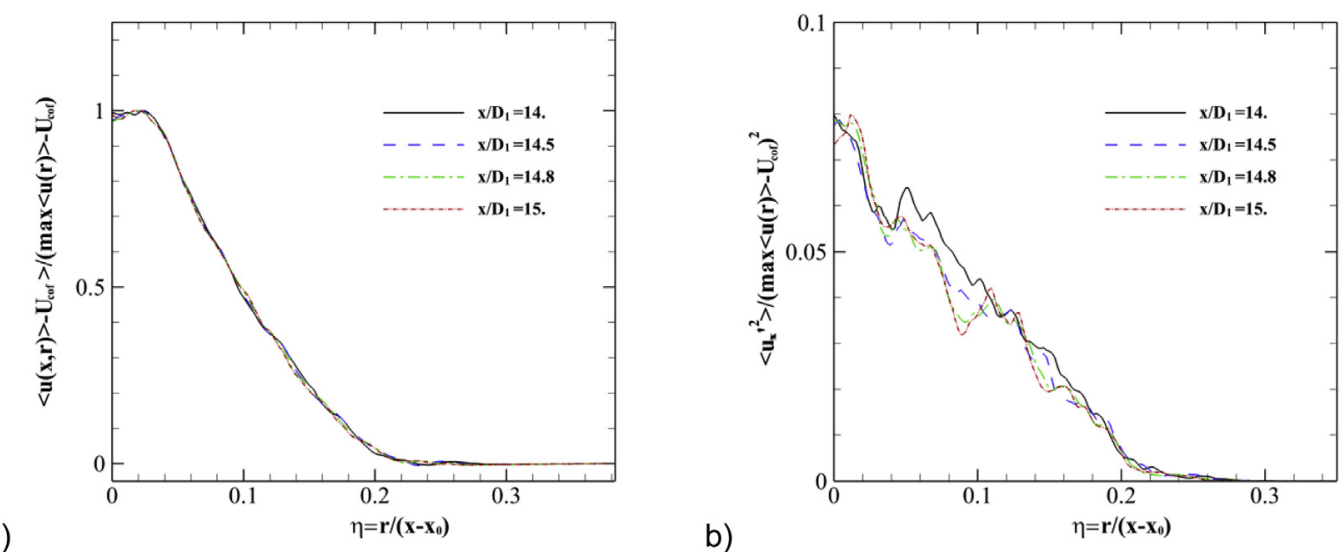

a)

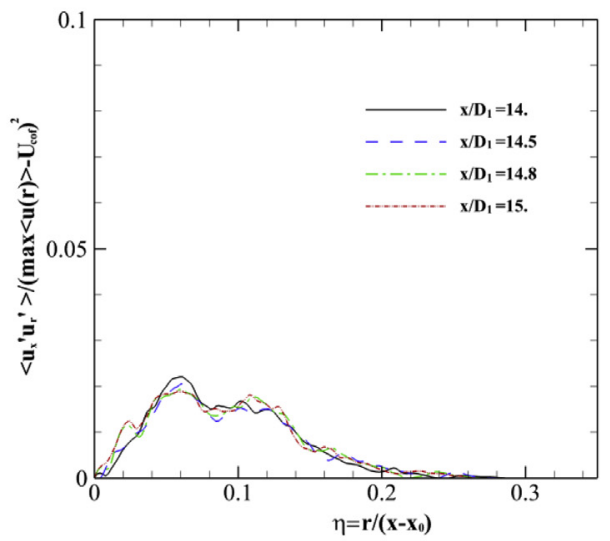

b)

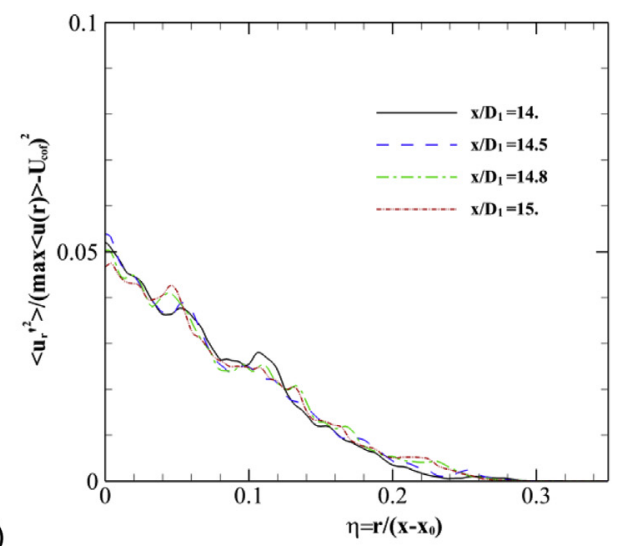

Fig. 21 - (a) Streamwise velocity profiles in the far-field of the non-isothermal jet where $U_{\text {cof }}$ is the local co-flow velocity and $x_{0}$ is the virtual jet origin, (b) streamwise normal Reynolds stresses, (c) streamwise radial stresses and (d) radial stresses.

The most striking effect of the temperature gradient is on the transition region as shown in Fig. 26a. Conversely to the previous case (isothermal jet), for $x / D_{1}=6$ at the center jet, PDF confirms that the turbulent mixing becomes more significant. We can clearly see the presence of the spots of mixing in the inner jet. These spots are the signatures of the inner Kelvin-Helmholtz vortices which play a more significant role in the turbulent mixing and enhance entrainment. Furthermore, at the end of domain computational, in the center jet the mixture fraction seems much better distributed, where the most likely value of mixture fraction is situated between 0.5 and 0.3 (Fig. 26b). In addition, the mixing seems also homogenously scattered near the jet center at this downstream location. This is a direct consequence of the precocious development Kelvin-Helmholtz vortices in the inner jet which plays a pivotal role in the mass transfer.

In order to get more insight of the mixing homogeneity beyond the end of the outer potential core, it would be instructive to examine the intensity of segregation quantity. This quantity, introduced by Danckwerts [50] is defined by: a)

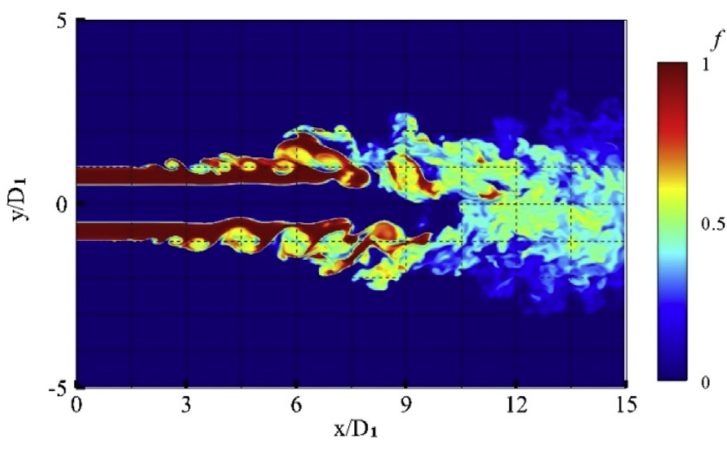

b)

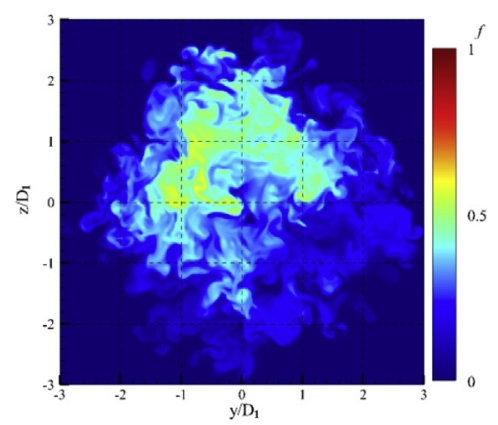

Fig. 22 - Instantaneous contours of mixture-fraction for the non-isothermal case: (a) in the central plane and (b) in the transverse section located at $x / D_{1}=14$. 
a)

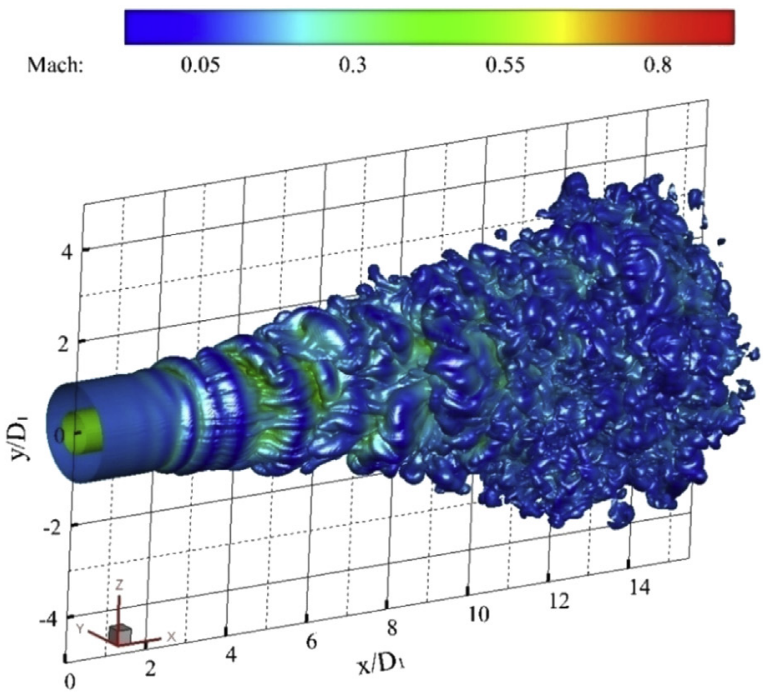

b)

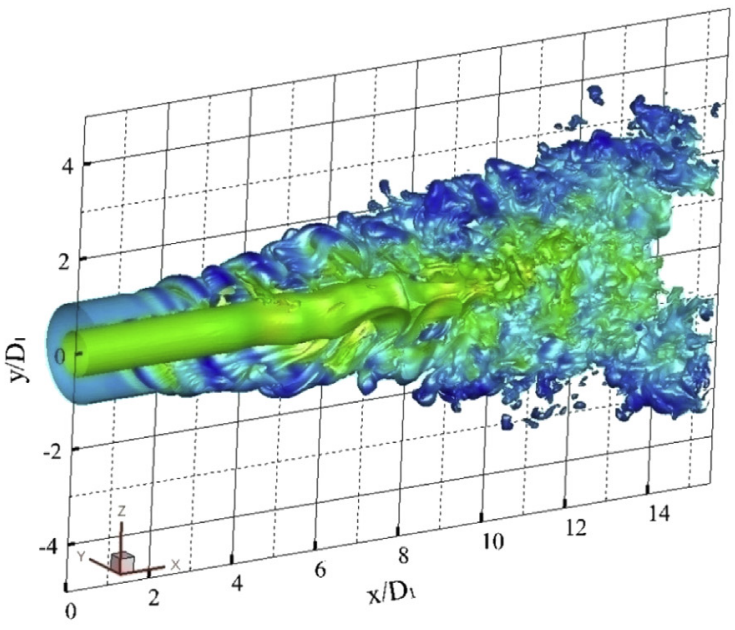

Fig. 23 - Global view of the non-isothermal jet case: (a) isosurface of mixture-fraction. (b) Cut view of $f=0.15$ mixture-fraction isosurface coloured by the local Mach number.

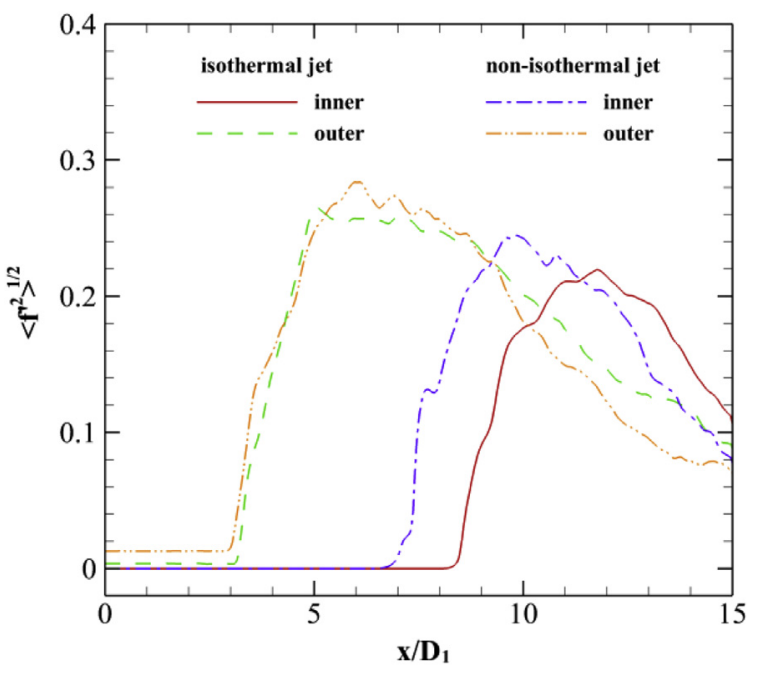

Fig. 25 - Comparison of the r.m.s mixture-fraction between the non-isothermal and the isothermal cases: downstream evolution in the inner $\left(r / D_{1}=0\right)$ and outer $\left(r / D_{1}=0.75\right)$ jets.

$$
I=\frac{<f^{\prime 2}>}{<f>(1-<f>)}
$$

This quantity means that the mixing becomes perfectly homogeneous when $I=0$, in contrast, when $I=1$ this means the species remain segregated.

Fig. 27 shows the radial distribution of the intensity of segregation in the non-isothermal jet at several downstream locations. Fig. 27 shows a good homogeneity at the center jet in this case at the end of the computational domain. For the transition region, $I=1$ becomes larger at $r / D_{1}<0.5$ (jet center) and at $r / D_{1}>2$ (interface between the jet and the ambient fluid); this indicates that the species are stirred but remains weakly segregated in that flow region which makes the mixing between species is the inhomogeneously scattered.

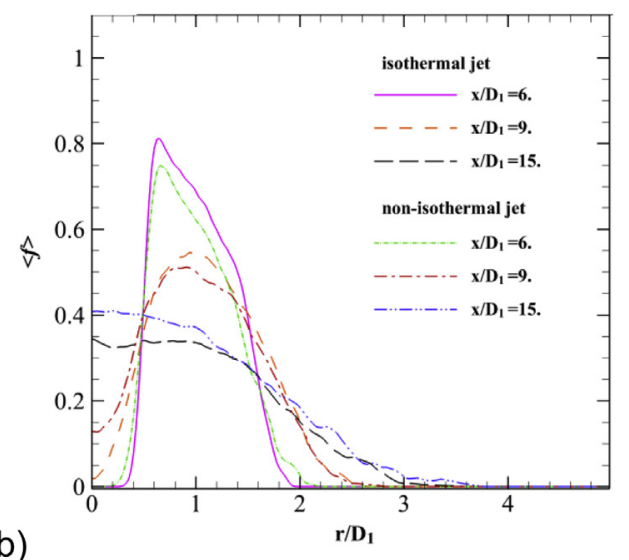

Fig. 24 - Comparison of the mean mixture-fraction between the non-isothermal and isothermal cases: (a) downstream evolution in the inner $\left(r / D_{1}=0\right)$ and outer $\left(r / D_{1}=0.75\right)$ jets; (b) profiles at several downstream locations. 


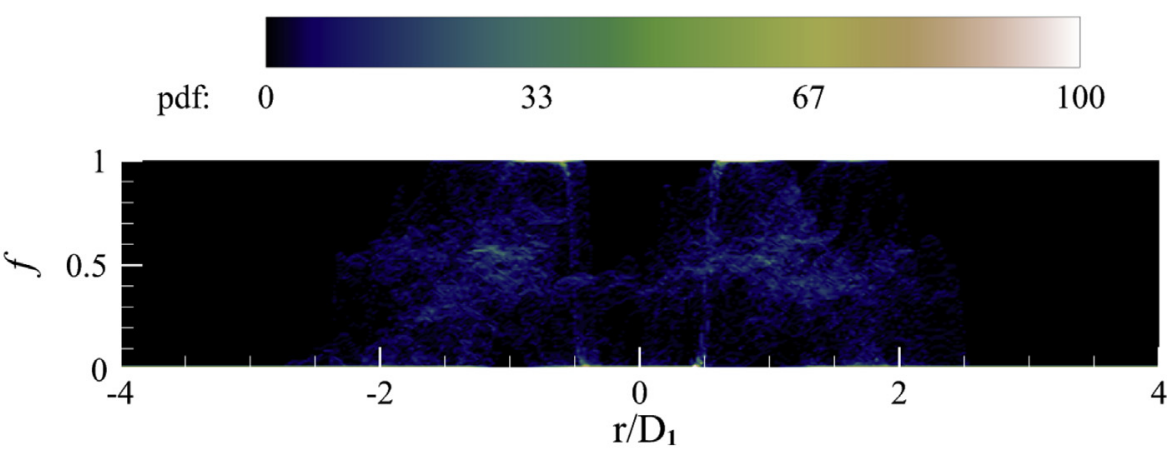

a)

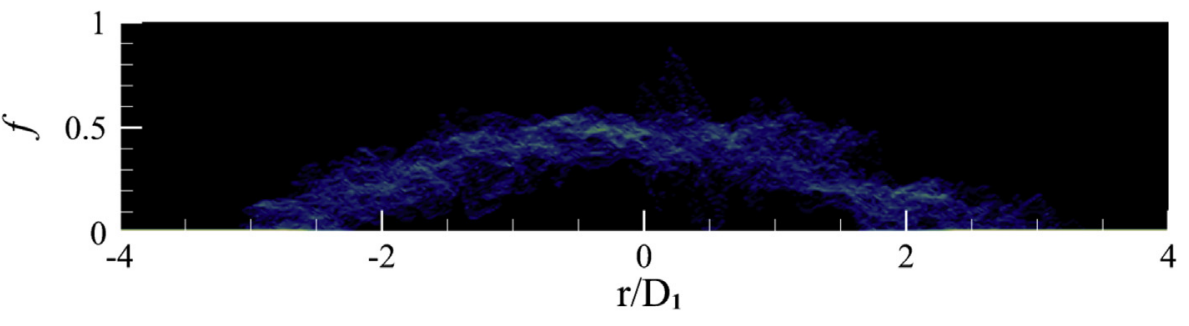

Fig. 26 - Variation of the mixture-fraction PDFs for the non-isothermal jet at: (a) $x / D_{1}=6$ and (b) $x / D_{1}=15$.

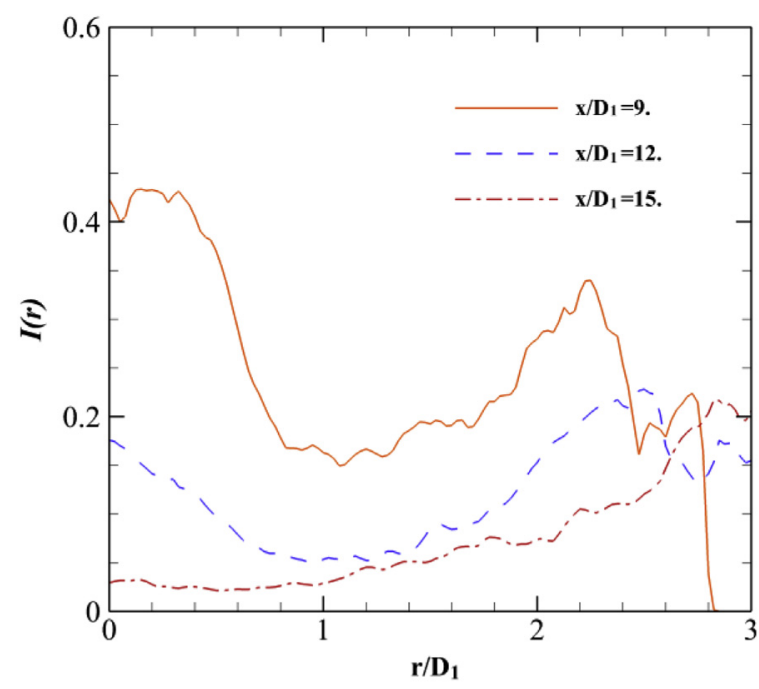

Fig. 27 - Radial evolution of the intensity of segregation at several downstream locations.

\section{Conclusion}

We have simulated numerically the spatial development of air-air compressible coaxial jets. The three-dimensional Monotone Integrated Large Eddy Simulations (MILES) approach is used to represent all the features of the turbulent mixing physics.

Two computations have been carried out to assess the effect of the temperature gradient (isothermal and nonisothermal coaxial jets) upon the development of coherent vortices, and hence on the mixing process.

The mixing between inner and outer jets is followed through the spatio-temporal evolution of the mixture fraction field. The spatially developing approach allows to take into consideration the different stages of the turbulent mixing: molecular diffusion, transition and fully developed turbulent state.

The computational simulations performed in this work reproduce qualitatively the flow structures and give satisfactory results in good agreement with the experimental data.

It was found that the inner potential core length of the coaxial jets depends strongly on the temperature gradients whereas the outer potential core length seems to be insensitive to the temperature gradients. Another major effect of temperature gradients is the enhancement threedimensionality of the jets.

Furthermore, a faster transition was observed in the nonisothermal case since inner streamwise vortices are formed earlier and so the transition phenomenon of the inner species is faster as compared to the isothermal case.

The mixing process is largely controlled by the dynamics and interactions of the structures vortices when the coaxial jet possesses a hot primary jet.

The analysis of mixture fraction fluctuations indicated that turbulent mixing activity begins to grow earlier in the nonisothermal case. This is in good agreement with the precocious development of the Kelvin-Helmholtz vortices.

At the exit of the computational domain, the mixture fraction PDFs distributions have shown that turbulent mixing becomes more homogeneous with the disappearance of spots of unmixed species in the case of the non-isothermal jet.

The results highlight also that the pronounced temperature gradient in the coaxial jet leads to precocious development of inner Kelvin-Helmholtz vortices, consequently, the enhancement of the turbulent mixing efficiency can be quite helpful in numerous industrial applications such as combustion, chemical engineering process and aeroacoustics.

The results obtained in this study can be regarded as a significant reference in active control of coaxial jets mixing, which will be studied in a forthcoming paper. 
[24] Balarac G, Métais O, Lesieur M. Mixing enhancement in coaxial jets through inflow forcing: a numerical study. Phys Fluids 2007;19:075102. 1-17.

[1] Narasimha Reddy NL, Manivannan P, Kiran Babu KM. CFD analysis of turbulence characteristics in combustion chamber with non circular co-axial jets. IOSR J Mech Civ Eng 2013;6(2):1-10.

[2] Seong RK, Wolfgang S, Matthias M. Noise sources in heated coaxial jets. Comput Fluids 2013;78:24-8.

[3] Bogey C, Barré S, Juvé D, Bailly C. Simulation of a hot coaxial jet: direct noise prediction and flow-acoustics correlations. Phys Fluids 2009;21(3):035105.

[4] Dimitri P, Andrew DJ, Vincent P. Aeroacoustics of threestream high-speed jets from coaxial and asymmetric nozzles. J Propul Power 2014;30(4):1055-69.

[5] Da Silva CB, Balarac G, Metais O. Transition in high velocity ratio coaxial jets analysed from direct numerical simulations. J Turbul 2003;4(24):1-18.

[6] Balarac G, Metais O. The near field of coaxial jets: a numerical study. Phys Fluids 2005;17:065102. 1-14.

[7] Balarac G, Si-Ameur M, Métais O, Lesieur M. Direct numerical simulations of high velocity ratio coaxial jets: mixing properties and upstream conditions influence. J Turbul 2007;8(22).

[8] Balarac G, Si-Ameur M. Mixing and coherent vortices in turbulent coaxial jets. C R Mecanique 2005;333:622-7.

[9] Zhdanov V, Hassel E. Mixing enhancement in a coaxial jet mixer. Adv Mater Phys Chem 2012;2:134-7.

[10] Liu Hai-TaoShi Nan-Sheng, Wang Pei, Lu Xi-Yun. Large-eddy simulation of sonic coaxial jets with different total pressure ratios of the inner to outer nozzle. Comput Fluids 2018;171:122-34.

[11] Cai J, Dinger MJ, Li W, Carter CD, Ryan MD, Tong C. Experimental study of three-scalar mixing in a turbulent coaxial jet. J Fluid Mech 2011;685:495-531.

[12] Villermaux E, Rehab H. Mixing in coaxial jets. J Fluid Mech 2000;(425):161-85.

[13] Rehab H, Villermaux E, Hopfinger EJ. Flow regimes of largevelocity ratio coaxial jets. J Fluid Mech 1997;345:357-81.

[14] Ranga-Dinesh KKJ, Savill AM, Jenkins KW, Kirkpatrick MP. A study of mixing and intermittency in a coaxial turbulent jet. Fluid Dynam Res 2010;42:025507.

[15] Montagnani D, Auteri F. Non-modal analysis of coaxial jets. J Fluid Mech 2019;872:665-96.

[16] Khodadadi JM, Vlachos NS. Experimental and numerical study of confined coaxial turbulent jets. AIAA J 1989;27(5):532-41.

[17] Fan J, Zhao H, Cen K. Numerical modeling and experimental study of particle-laden coaxial jets. Chem Eng Commun 1989;86:55-71.

[18] Nikjooy M, Karki KC, Mongia HC. A numerical and experimental study of coaxial jets. Int J Heat Fluid Flow 1989;10(3):253-61.

[19] Forstall W, Shapiro AH. Momentum and mass transfer in coaxial gas jets. J Appl Mech 1951;18:219-23.

[20] Ko NWM, Kwan ASH. The initial region of subsonic coaxial jets. J Fluid Mech 1976;73:305-32.

[21] Kwan ASH, Ko NWM. The initial region of subsonic coaxial jets: part 2. J Fluid Mech 1977;82:273-87.

[22] Dahm WJA, Friedler CE, Tryggvason G. Vortex structure and dynamics in the near field of a coaxial jet. J Fluid Mech 1992;241:371-402.

[23] Champagne FH, Wygnanski IJ. An experimental investigation of coaxial turbulent jets. Int J Heat Mass Tran 1971;14:1445-64.

[25] Saravanan G, Vinoth Kumar A, Ravichandrakumar KB. Numerical analysis of subsonic coaxial jet on effect of potential core length. J Adv Mech Eng Sci 2016;2(1):26-32.

[26] Reynier P, Minh HH. Numerical prediction of unsteady compressible turbulent coaxial jets. Comput Fluids 1998;27(2):239-54.

[27] Ouzani R, Si-ameur M. Numerical study of hydrogen-air mixing in turbulent compressible coaxial jets. Int J Hydrogen Energy 2015;40:9539-54.

[28] Erina M, Dimitri P. Mean flow development in dual stream compressible jets. AIAA J 2002;40(6):1131-8.

[29] Guitton A, Tinney CE, Jordan P, Delville J. Measurements in a co-axial subsonic jet. In: 45th AIAA aerospace sciences Meeting and exhibit, Reno, NV; 2007.

[30] Guitton A. Etude expérimentale des relations entre les champs hydrodynamiques et acoustiques des jets libres. Thèse de Doctorat. Université de Poitiers; 2009.

[31] Hashimoto T, Koyama K, Yamagishi M. Hydrogen combustion characteristics in a model burner with a coaxial injector. Int J Hydrogen Energy 1998;23(8):713-20.

[32] Shudo T, Omori K, Hiyama O. NOx reduction and NO2 emission characteristics in rich-lean combustion of hydrogen. Int J Hydrogen Energy 2008;33:4689-93.

[33] Tabet F, Sarh B, Gokalp I. Hydrogenehydrocarbon turbulent non-premixed flame structure. Int J Hydrogen Energy 2009;34:5040-7.

[34] Hashemi SA, Fattahi A, Sheikhzadeh GA, Mehrabian MA. Investigation of the effect of air turbulence intensity on NOx emission in non-premixed hydrogen and hydrogenhydrocarbon composite fuel combustion. Int J Hydrogen Energy 2008;33:4689-93.

[35] Oh J, Yoon Y. Flame stabilization in a lifted non-premixed turbulent hydrogen jet with coaxial air. Int J Hydrogen Energy 2010;35(19):10569-79.

[36] Kim TY, Choi S, Kim YH, Ahn YJ, Kim HK, Kwon OC. Combustion characteristics of gaseous inverse $\mathrm{O}_{2} / \mathrm{H}_{2}$ coaxial jet flames in a single-element model combustor. Energy 2018;155:262-71.

[37] Colella P, Woodward PR. The piecewise parabolic method (PPM) for gas-dynamical simulations. J Comput Phys 1984;54:174-201.

[38] Roe PL. Approximate Riemann solvers, parameter vectors and difference schemes. J Comput Phys 1981;43:357-72.

[39] Willamson JH. Low storage RungeKutta schemes. J Comput Phys 1980;35:48-56.

[40] Morris PJ. The spatial viscous instability of axisymmetric jets. J Fluid Mech 1976;77:511-29.

[41] Michalke A, Hermann G. On the inviscid instability of a circular jet with external flow. J Fluid Mech 1982;114:343-59.

[42] Hunt JCR, Wray AA, Moin P. Eddies, stream, and convergence zones in turbulent flows. In: Annual research briefs. Stanford: Center for Turbulence Research; 1988.

[43] Dubief Y, Delcayre F. On coherent vortex identification in turbulence. J Turbul 2000;1(11):1-22.

[44] Danaila I, Dusek J, Anselmet F. Coherent structure in a round, spatially evolving, unforced, homogeneous jet at low Reynolds number. Phys Fluids 1997;9:3323-42.

[45] Brancher P, Chomaz JM, Huerre P. Direct numerical simulations of round jets: vortex induction and side jets. Phys Fluids 1993;6:1768-74.

[46] Stanley SA, Sarkar S, Mellado JP. A study of the flow-field evolution and the mixing in a plane turbulent jet using direct numerical simulation. J Fluid Mech 2002;450:377-407. 
[47] Lau JC. Effect on exit Mach number and temperature on mean flow and turbulence characteristics in round jet. J Fluid Mech 1981;105:193-208.

[48] Bodony DJ, Lele SK. On using large-eddy simulation for the prediction of noise from cold and heated turbulent jets. Phys Fluids 2005;17:085103.
[49] Hussein HJ, Capp SP, George WK. Velocity measurements in a high-Reynolds-number, momentum-conserving, axisymmetric, turbulent jet. J Fluid Mech 1994;258:31-75.

[50] Danckwerts PV. The definition and measurement of some characteristics of mixture. Appl Sci Res, Sect A 1952;3:279. 